\title{
Le décloisonnement du passage du Nord-Ouest
}

Le tourisme de croisière en éclaireur avant l'arrivée des pétroliers et autres grands transporteurs

The opening of the Northwest Passage: Cruise tourism as a scout before the arrival of tankers and other major carriers

A abertura da Passagem do Noroeste: o turismo de cruzeiro como precursor, antes da chegada dos petroleiros e dos grandes navios transportadores

\section{Alain Adrien Grenier}

\section{(2) OpenEdition}

\section{Journals}

Édition électronique

URL : https://journals.openedition.org/ideas/3313

DOI : $10.4000 /$ ideas.3313

ISSN : 1950-5701

Éditeur

Institut des Amériques

Référence électronique

Alain Adrien Grenier, «Le décloisonnement du passage du Nord-Ouest », IdeAs [En ligne], 12 | 2018, mis en ligne le 19 novembre 2018, consulté le 19 octobre 2022. URL : http://journals.openedition.org/ ideas/3313 ; DOI : https://doi.org/10.4000/ideas.3313

Ce document a été généré automatiquement le 19 octobre 2022.

\section{c)}

Creative Commons - Attribution - Pas d'Utilisation Commerciale - Pas de Modification 4.0 International - CC BY-NC-ND 4.0

https://creativecommons.org/licenses/by-nc-nd/4.0/ 


\section{Le décloisonnement du passage $d u$ Nord-Ouest}

Le tourisme de croisière en éclaireur avant l'arrivée des pétroliers et autres grands transporteurs

The opening of the Northwest Passage: Cruise tourism as a scout before the arrival of tankers and other major carriers

A abertura da Passagem do Noroeste: o turismo de cruzeiro como precursor, antes da chegada dos petroleiros e dos grandes navios transportadores

Alain Adrien Grenier

Remerciements

L'auteur tient à remercier François Weigel, chargé de mission recherche/édition, Institut des Amériques, Vincent Andreu-Boussut, maître de conférences en géographie, Le Mans Université et deux réviseurs anonymes pour des commentaires sur une version préliminaire du manuscrit.

\section{Introduction}

1 Le passage du Nord Ouest est un corridor maritime qui sépare d'ouest en est le continent nord-américain de l'archipel arctique (illustration 1). La traversée des 2400 $\mathrm{km}$ du passage, de l'île Banks, à l'ouest, à la Terre de Baffin, à l'est, fut longtemps impossible à réaliser en raison des glaces qui y perduraient même l'été. En dépit de la perte de plusieurs explorateurs, le passage du Nord-Ouest a toujours attiré l'attention de groupes d'intérêts qui voient en lui la promesse d'une voie de navigation plus courte et plus profitable pour le transport des biens et marchandises entre l'Europe et l'Asie. 


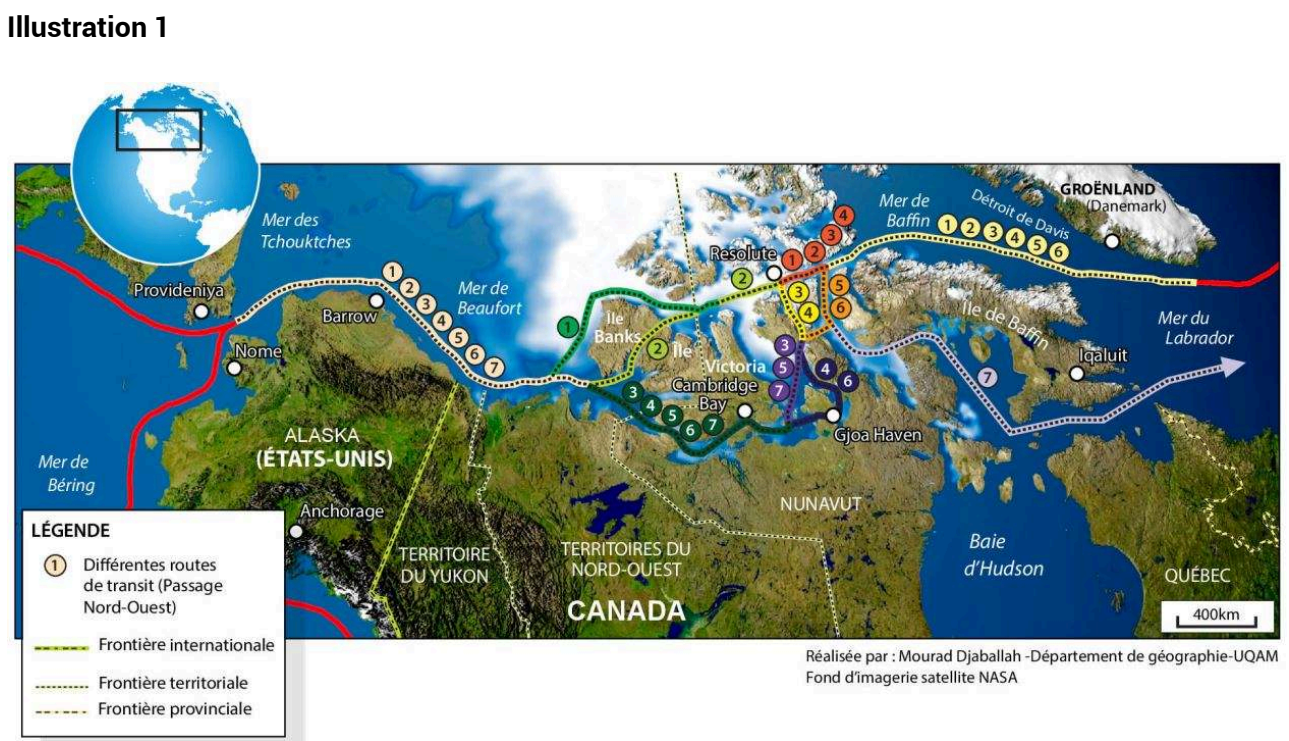

LE PASSAgE DU NORD-OUEST, ENTRE LE CONTINENT NORD-AMÉRICAIN, ET L'ARCHIPEL ARCTIQUE.

Source : NASA (domaine public)

2 Or, depuis près de deux décennies, les signes du réchauffement climatique dans l'Arctique canadien, et plus précisément dans la zone constituée par le passage du Nord-Ouest, ne se démentent pas. Les études (Howell et al., 2007; Wilson et al., 2004) et les observations des Inuits (Guérin-Lajoie et al., 2016) qui y vivent le confirment : depuis 1979, la banquise décroît de façon importante (3.7 \% par décennie), tant en surface couverte qu'en épaisseur (Arctic Council, 2009 : 25). La fonte de la glace, plus ou moins rapide, selon les secteurs (Huebert, $2011: 383$ ), fait miroiter la possibilité d'un passage du Nord-Ouest plus accessible (Howell et al., $2007: 229$ ), et même libre de glaces, de 2 à 4 mois, l'été, autour de 2100 (Arctic Council, 2009 : 27; Khon et al., 2010 : 757), ou même avant. De là, la mise à jour du rêve de transit via le passage du Nord-Ouest. Cet enthousiasme a été déclaré haut et fort par des groupes d'intérêt (armateurs et États d'Asie) en octobre 2013 à Reykjavik, en Islande, à l'occasion du premier forum de l'organisation Arctic Circle, lancée à l'initiative de son président, Ólafur Ragnar Grímsson. Le forum réunit les voix concernées par les changements auxquels font face les diverses régions de l'Arctique ${ }^{1}$. Le désir avoué de ces groupes d'intérêts de recourir dans un avenir proche au passage du Nord-Ouest pour le transport des cargaisons, défie du coup ouvertement la souveraineté du Canada sur ce passage qu'il considère comme faisant partie de ses eaux intérieures.

3 Le Canada a pourtant du mal à affirmer sa souveraineté : 1) il ne dispose d'aucune infrastructure portuaire (à l'exception de celles du village de Toktoyaktuk);2) les bases militaires chargées de la surveillance de l'Arctique sont situées à une journée de vol, dans le sud du Canada ; 3) la flotte vieillissante de brise-glaces de la Garde côtière canadienne nécessite d'être remplacée ; et 4) la route navigable n'est cartographiée que sur un petit corridor, entre les villages de Cambridge Bay et Gjoa Haven. Ces éléments ont de quoi inquiéter. L'Arctique canadien est partagé de part et d'autre du passage du Nord-Ouest. Il est morcelé en détroits et chenaux parsemés d'îles de toutes les tailles (plus de 36000 îles, seulement pour son archipel). Il constitue un véritable terrain d'obstacles pour la navigation, quand les conditions sont à leur meilleur. Plusieurs navires l'ont récemment appris à leurs dépens en s'échouant sur des hauts fonds. 
Devant la prudence des compagnies pétrolières et des armateurs, ce sont les touristes qui, avançant vers l'inconnu, défient les risques, déployant ainsi argent et efforts pour atteindre et explorer ces régions méconnues du reste du monde. Sans aucune garantie de pouvoir passer les glaces, ils s'aventurent à la recherche des ressources naturelles (paysages, faune et flore) et culturelles (populations inuites) promises par les voyagistes, assoiffés du désir de faire reculer l'impossible du voyage d'agrément. Ce tourisme polaire, qui se veut non intrusif, contribue inévitablement au décloisonnement de l'Arctique et de son peuple inuit. Car en négociant ses traversées à travers les glaces, en explorant chacune des voies navigables qui composent le passage $\mathrm{du}$ Nord-Ouest, l'industrie des croisières polaires trace la voie, sans le vouloir, à d'autres groupes d'intérêts qui n'auront pas forcément à cœur ceux des Inuits et de leur environnement fragile.

C'est dans ce contexte que la présente étude montre comment l'industrie de la croisière polaire, par son exploration des eaux arctiques, contribue au décloisonnement d'un territoire qu'elle cherchait, a priori, à protéger. Pour soutenir son argument, l'étude :

- - a) recense les intérêts autour de la navigation commerciale du passage du Nord-Ouest;

- b) illustre comment l'industrie de la croisière polaire construit un espace touristique à l'intérieur du passage du Nord-Ouest;

- - c) discute des enjeux liés à la navigation dans ce corridor maritime dans l'état actuel des connaissances sur le milieu.

\subsection{Méthodologie}

6 L'étude adopte une approche qualitative de type phénoménologique. La phénoménologie construit son analyse autour de l'expérience des sujets mais aussi de celle du chercheur. Ainsi, outre les références à des études déjà publiées sur le sujet, l'article s'appuie essentiellement sur des observations du chercheur réalisées dans de multiples régions de l'Arctique circumpolaire et du Nord (Amérique, Europe, Asie). À cela s'ajoutent des enquêtes réalisées à bord de navires de croisières à l'occasion de trois traversées sectorielles du passage du Nord-Ouest $(1999,2010,2013)$. Durant ces séjours, le chercheur a eu accès à la passerelle de navigation de chacun des navires (à l'exception des accostages dans les ports). Il a ainsi pu observer le travail des navigateurs et les interroger, lorsque cela était possible, sur des questions relatives à la navigation en eaux polaires. La consultation des itinéraires des voyagistes à partir de leurs sites Internet, complète les informations sur les produits touristiques lorsque nécessaire.

\section{Géographie et ouverture commerciale d'un passage mythique}

\subsection{Géographie}

7 L'Arctique est un concept géoculturel qui se définit tantôt selon des considérations géopolitiques (frontières et distances des centres administratifs du Sud), économiques (dépendance de l'économie aux centres administratifs du Sud), géophysiques (constitution et histoire géologique, axe de rotation de la Terre avec le cercle polaire), 
climatiques (ligne des arbres et ligne du pergélisol, ligne d'isotherme et zones climatiques) et culturelles (les perceptions que s'en font les gens dans leur imaginaire) ${ }^{2}$.

8 L'Arctique canadien s'étend d'Ouest en Est des plaines septentrionales du Yukon, à la frontière de l'Alaska, jusqu'aux montagnes de l'Ungava, à cheval sur la frontière du Québec et du Labrador. Du Sud, il va de la Baie d'Hudson jusqu'à 75 degrés de latitude Nord, à quelques heures de navigation du Pôle Nord $\left(90^{\circ} \mathrm{N}\right)$. Sa principale partie se trouve dans l'État du Nunavut où vivent 38000 habitants répartis inégalement sur un territoire de $2038722 \mathrm{~km}^{2}$ (terrestre et maritime). Les Inuits constituent l'ethnie principale de l'Arctique canadien, établie préalablement à la colonisation du territoire par les Européens. Sédentarisés, leur mode de vie compte encore néanmoins sur certaines traditions de chasse (y compris la trappe) et de pêche, pratiquées avec des équipements modernes (fusil, motoneige, etc.), bien que le kayak soit encore employé. L'hiver, les Inuits se déplacent par les détroits et autres chenaux gelés du passage. Les navigateurs doivent veiller pendant les traversées à ne pas briser les routes de glace des Inuits qui pourraient ainsi se voir incapables de regagner leurs villages. La chasse à la baleine est toujours d'actualité pour ce peuple qui s'approvisionne principalement dans les détroits de Barrow et de Lancaster (Pharand, 2007: 21), aujourd'hui visés par la navigation commerciale dans le passage du Nord-Ouest.

Encore méconnu à bien des égards, l'Arctique a tendance à être perçu par le public comme une immense région uniforme. Ce n'est pas le cas: l'Arctique canadien se caractérise par des régions tout aussi différentes les unes des autres sur les plans géographique, climatique, faunique, floral et humain. Il faut savoir que l'Arctique canadien est composé de terres et de bassins marins subdivisés en quatre (4) grandes régions administratives - Inuvialuit, le Nunavut, le Nunaviket Nunatsiavut (illustration 2). Le Nunavut est à son tour subdivisé en trois (3) régions administratives - Kitikmeot, Kivalliq et Qikiqtaaluk. Ainsi, le développement commercial du passage du Nord-Ouest chevauche six (6) régions administratives, à savoir (d'Ouest en Est):

- - 1) Inuvialuit (versant pacifique ou Arctique de l'Ouest, Territoires du Nord-Ouest);

- - 2) Kitikmeot (centre de l'Arctique, Nunavut) ;

- - 3) Kivalliq (Nunavut);

- - 4) Qikiqtaaluk (archipels de Baffin et du Haut Arctique, Nunavut);

- 5) Nunavik (Nord du Québec);

- - 6) Nunatsiavut (Nord de Terre-Neuve-et-Labrador). 


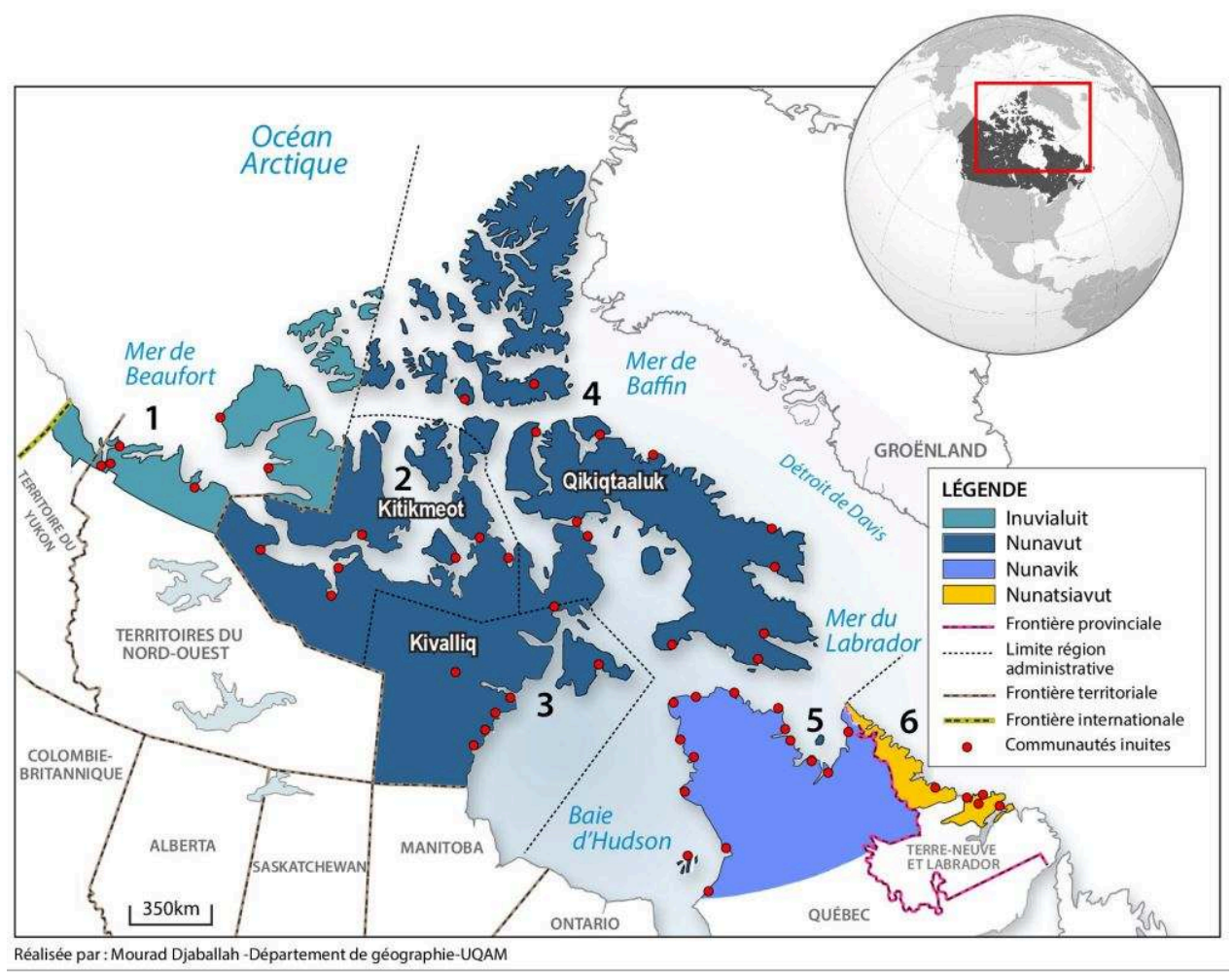

LES QUATRE RÉgIONS INUITES dU GRAND NORD CANADIEN

Source : Modifié par l'auteur à partir de Inuit Tapiriit Kanatami (2005).

Sur le plan géologique, la partie ouest du passage est composée de hauts plateaux aux versants extrêmement abrupts qui rappellent l'étendue aplanie des prairies canadiennes, plus au sud. En revanche, l'Arctique de l'Est (Terre de Baffin et île d'Ellesmere) est dentelée par la présence de la Cordillère Arctique - une vaste chaine de montagnes très accidentées qui prend naissance dans l'Ungava, à la frontière du Québec et du Labrador. Plusieurs sommets dépassent les $2000 \mathrm{~m}$, dont le mont Barbeau, sur l'île d'Ellesmere, L'île d'Ellesmere se caractérise par d'imposantes montagnes, des fjords et des glaciers qui se jettent dans la mer. En raison des nombreux canaux qui séparent les îles de l'archipel, la glace hivernale a tendance à s'accumuler dans cette région, la rendant très difficile d'accès par la mer. En retour, elle constitue un environnement idéal pour certaines espèces fauniques arctiques. Sur le plan écosystémique, les conditions de salinité très différentes entre les océans externes (Pacifique et Atlantique) et les bassins internes de l'Arctique affectent la répartition inégale des espèces marines (ours polaires, phoques et baleines). Ensembles, la géologie et la distribution des espèces animales, deux attraits majeurs pour le tourisme, influenceront la planification des itinéraires de croisière.

\subsection{Un passage commercial}

11 Les avantages de la navigation commerciale via le passage du Nord-Ouest, la libération des glaces advenant au moins une partie de l'année, sont indéniables. En évitant aux navires de passer par le canal de Panama, le passage du Nord-Ouest permettrait de réduire de $8000 \mathrm{~km}$ la distance actuelle entre l'Asie et la côte est des États-Unis. En 
1969, le pétrolier américain SS Manhattan a démontré qu'il était possible pour un navire de 120000 tonnes de traverser (sans autorisation) le passage entier.

Entre la première traversée complète du passage du Nord-Ouest (1903-1906) et 2017, plus de 287 navires ont réussi à voyager d'un océan à l'autre via le passage du NordOuest (Headland, 2018 ${ }^{3}$ ). De ce nombre, plus de la moitié des traversées ont été réalisées par des navires de plaisance (56 navires de croisière, 118 yachts et une vingtaine de voiliers). Les autres ont été réalisées par des brise-glaces dont plusieurs battant pavillon étranger, au moins 3 pétroliers et un baleinier.

Plusieurs pays non liés à l'Arctique entrevoient avec optimisme l'occasion de diversifier leur économie et d'assurer leur croissance économique par l'entremise du passage du Nord-Ouest. Ils le voient à la fois comme route de navigation commerciale mais aussi comme lieu d'extraction des ressources (Bennett, 2016). La disparition des glaces facilitera également l'exploitation des réserves de pétrole et gaz naturels dans cette région du monde (Huebert, 2011 : 385). Ce n'est pas par un hasard si cinq pays d'Asie (la Chine, l'Inde, le Japon, la Corée du Sud et Singapour) ont fait leur entrée en 2013 au sein du Conseil de l'Arctique - un forum créé à l'origine par et pour les pays de l'Arctique. Chacun de ces pays développe ainsi des initiatives liées à l'avenir de l'Arctique canadien. La Chine aspire même à devenir un «État nordique » (Hopper, 2018). Elle a ainsi dépêché un navire dans les eaux du passage en 2012 afin d'y recueillir des données sur la navigation (Fife, 2017). Cette situation ne réjouit pas le Canada qui revendique la souveraineté sur l'ensemble du passage du Nord-Ouest - une position que ne reconnaissent ni les États-Unis, ni l'Union européenne (Huebert, 2011 : 388).

Actuellement, tout navire qui entre dans les eaux du passage du Nord-Ouest est invité à s'enregistrer auprès du Service de trafic maritime nordique canadien (NORDGREG), géré par la Garde côtière canadienne à Iqaluit, capitale du Nunavut. Mais cet enregistrement se fait uniquement sur la base du volontariat, ce qui représente une difficulté supplémentaire pour l'État canadien dans l'affirmation de sa souveraineté sur cet espace maritime (Huebert, 2011: 395). Rendre l'enregistrement obligatoire nécessiterait pour le gouvernement canadien d'être capable d'arraisonner tous les intrus. Or, l'État canadien ne dispose pas des navires nécessaires pour y parvenir. En 1999, la visite non annoncée d'un navire de recherche chinois à Tuktoyaktuk a pris les villageois par surprise (Huebert, 2011 : 384). L'ambassade canadienne à Pékin avait été informée des intentions du navire mais l'information n'avait apparemment pas été relayée aux autorités locales, pas plus qu'à la Garde côtière qui a appris la nouvelle après le départ du navire (Huebert, $2011: 384$ ).

15 En 2013, alors que le navire à bord duquel je me trouvais tentait de franchir le passage du Nord-Ouest par le détroit de Bellot, nous y avons croisé un yacht privé (illustration 3), qui a refusé de s'identifier, exigeant plutôt que nous nous en éloignions, sans autre explication. Ce comportement a laissé notre équipage perplexe dans un environnement où l'entraide est de mise. Quelques heures plus tard, nous voyant rebrousser chemin, le yacht sollicita notre évaluation sur l'état des glaces dans le détroit (illustration 4). On apprit alors que l'équipage avait signé une entente de confidentialité et ne pouvait dévoiler l'identité ni même l'origine de son/ses passager(s). Quelques jours plus tard, alors que nous nous trouvions à l'île Pim $\left(78^{\circ} \mathrm{N}\right)$, notre navire a été surpris par un avion patrouilleur de la Garde côtière canadienne qui n'avait pas été avisé de notre présence. Tournoyant au-dessus de nous, l'appareil de reconnaissance a longtemps interrogé 
l'équipage en devoir, afin de savoir qui nous étions, les raisons d'être de notre présence, notre provenance, etc.

\section{Illustration 3}

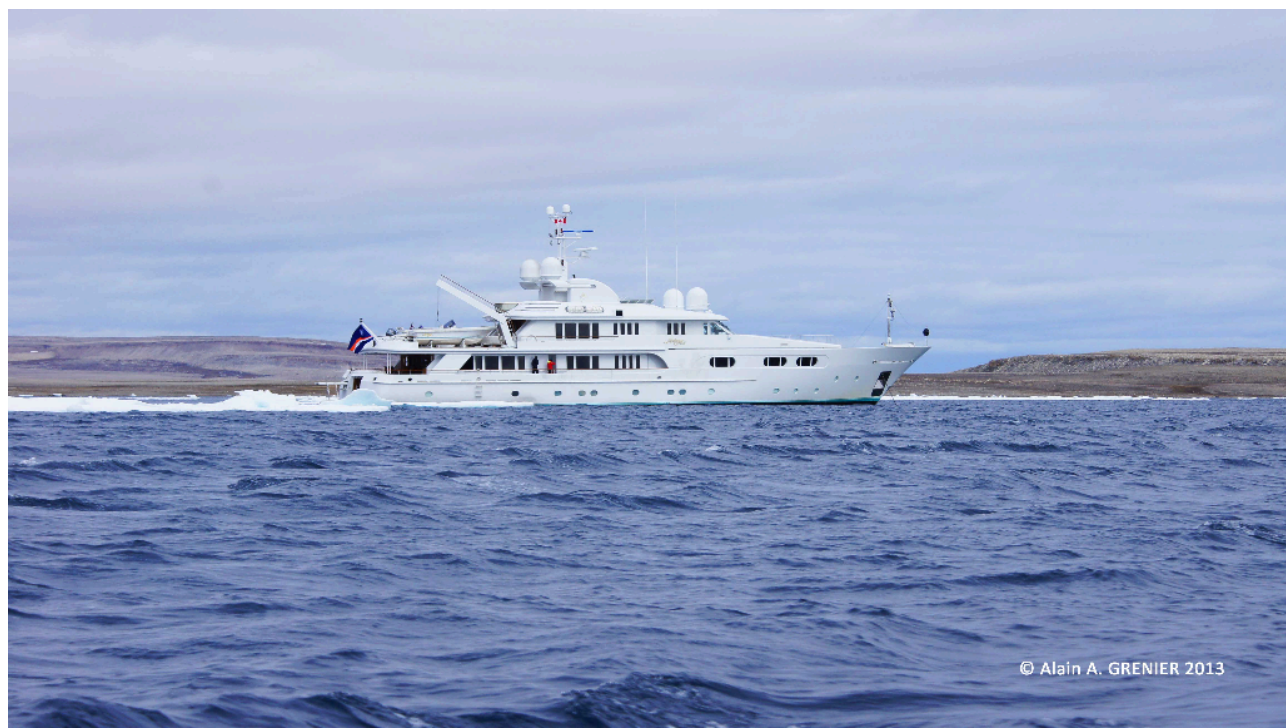

YACHT NON-IDENTIfiÉ CROISÉ À L'île SOMERSET (ARRIÈRE-PLAN), À L'ENTRÉE DU DÉTROIT DE BELLOT. L'ÉQUIPAgE, QUI DISAIT AVOIR SIgNÉ UNE ENTENTE DE CONfIDENTIALITÉ AVEC SON/SES PASSAgER(S), NOUS A DEMANDÉ DE NE PAS LES APPROCHER.

Source : Alain A. Grenier

\section{Illustration 4}

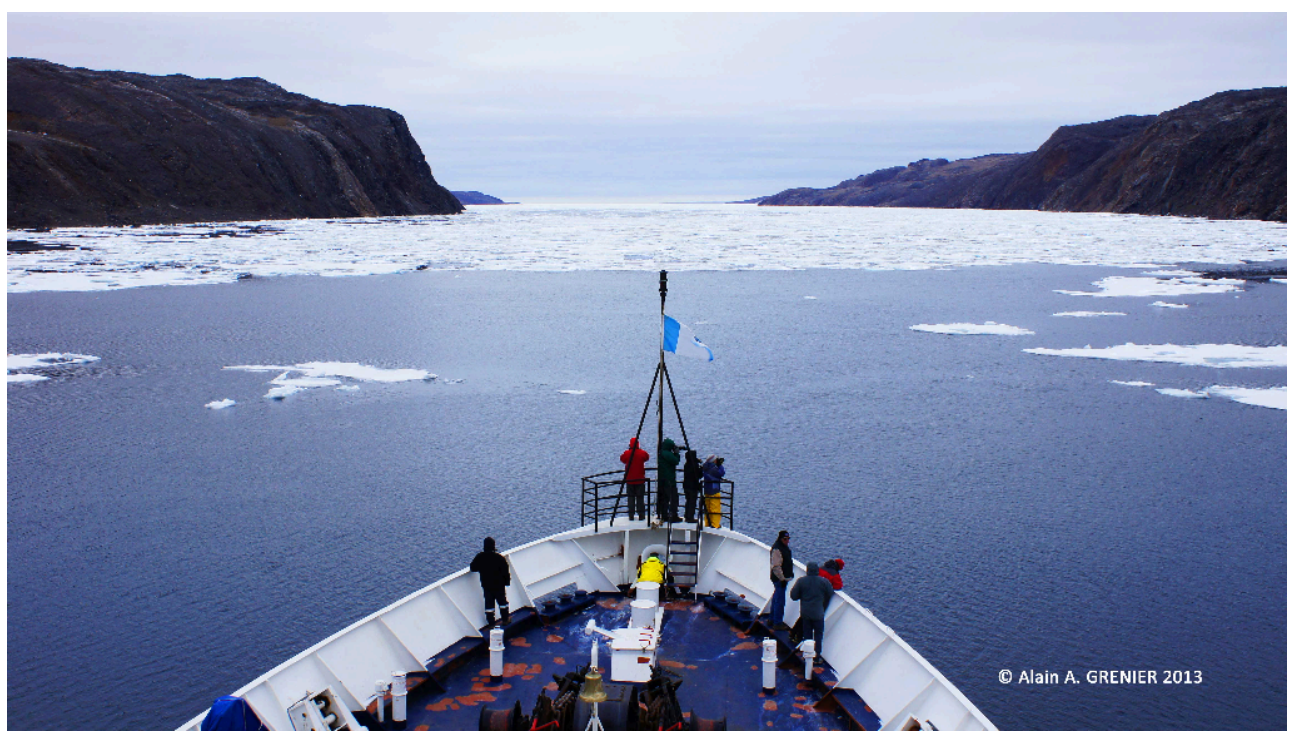

LE DÉTROIT DE BELLOT, EN DIRECTION OUEST, BLOQUÉ PAR LES gLACES.

Source : Alain A. Grenier

Bien qu'anecdotiques, ces épisodes montrent 1) qu'il est possible de pénétrer à l'intérieur du territoire arctique canadien sans autorisation, et 2) que l'État canadien assure une présence et arrive à détecter et documenter les intrusions. 
L'arrivée en grand nombre de navires dans le passage du Nord-Ouest aura inévitablement un impact. Les populations de l'Arctique, longtemps coupées du reste du monde, ont su utiliser leur isolement géographique pour protéger leurs cultures. La faune et la flore étaient elles aussi protégées par la barrière climatique. Or, les changements apportés par les modifications du climat amènent des espèces animales étrangères au milieu (comme l'orque) à concurrencer des espèces arctiques, jusque-là sans prédateur - comme le narval (voir Breed et al., 2017; Wisz et al., 2015; Kelly et al., 2010). Sur le plan sociopolitique, le décloisonnement de l'Arctique nord-américain va d'abord affecter les communautés inuites qui se trouvent sur son parcours - Cambridge Bay, Gjoa Haven, Resolute, Arctic Bay/Nanisivik, Pond Inlet, Clyde River Iqaluit et possiblement Repulse Bay et Cape Dorset. La fréquentation de leurs espaces par de grands navires perturbera leurs activités de chasse et de pêche (Huebert, 2011 : 385). Les contacts avec des marins en provenance majoritairement de pays du sud peuvent être la porte d'entrée de souches de maladie contre lesquelles les populations locales ne sont pas immunisées (Huebert, $2011: 396)$.

La commercialisation d'autres routes maritimes, dans le monde, démontre aussi le risque d'une augmentation de l'activité criminelle internationale - contrebande et braconnage notamment (Huebert, 2011 : 396). Des marins interrogés par le chercheur lors d'une traversée du passage du Nord-Est, en Russie (en 1998), ont confié qu'il n'était pas rare que des marins débarquent sur la banquise pour abattre un ours polaire (illustration 5) afin d'en revendre la peau ou les organes génitaux sur les marchés asiatiques où ils sont valorisés dans différentes pratiques médicinales. Au Canada, la valeur de la dépouille d'un ours polaire a quintuplé en dix ans, passant de 5000 \$ (CAD) en 2008 à 25000 \$ (CAD) en 2018 (Von Scheel, 2018).

\section{Illustration 5}

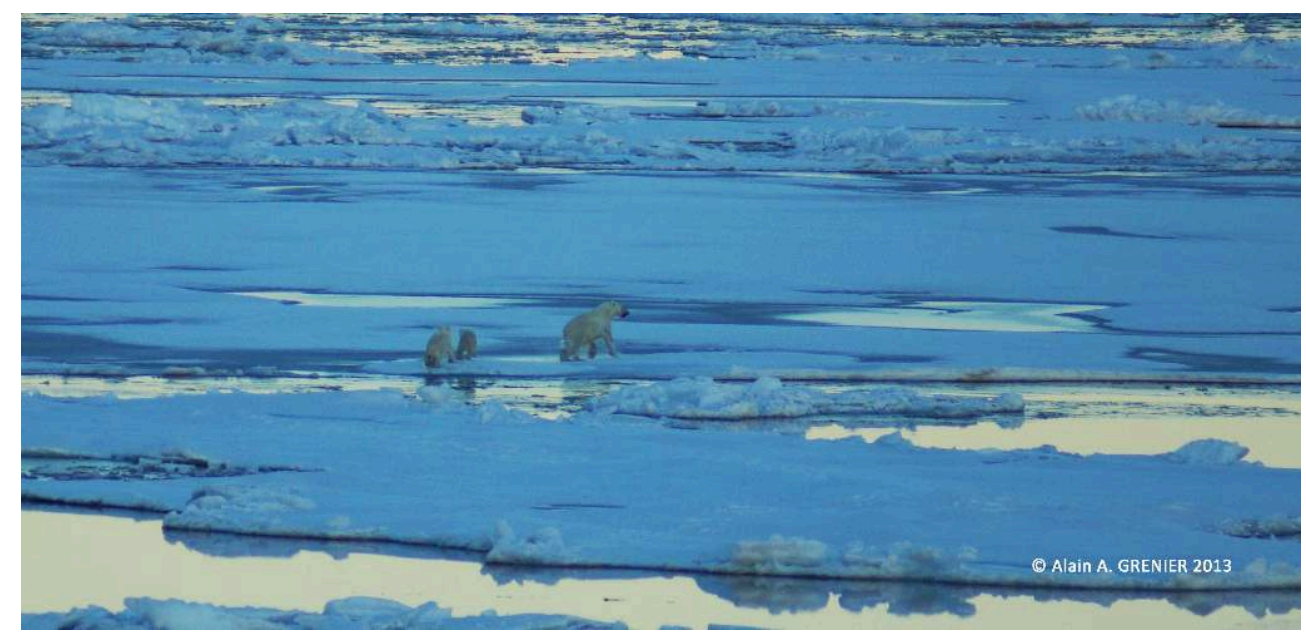

LES OURS POLAIRES, SUR LA BANQUISE, OU EN MER, SONT TRÈS VULNÉRABLES AU BRACONNAgE, LES NAVIRES ET LEURS ÉQUIPAgES ÉTANT PEU SI NON AUCUNEMENT SURVEILLÉS DANS CES LATITUDES ISOLÉES.

Source : Alain A. Grenier

En attendant la venue des pétroliers et autres cargos, c'est le tourisme qui pousse l'ouverture de cette voie maritime. Les passagers investissent des sommes colossales (plus de 10000 \$USD) pour atteindre le Haut Arctique afin d'y apprécier ses panoramas glacés, sa faune, sa flore et ses communautés inuites. L'industrie dit créer des 
«ambassadeurs" des pôles et croit ainsi sensibiliser ces visiteurs privilégiés à la nécessité de protéger l'Arctique.

\section{Tourisme nordique et polaire} la fois à la géographie, à la sociologie et à la culture. On peut le définir comme «toute activité ou expérience de séjour, quelle que soit la région, qui fait appel ou qui évoque la mythologie des milieux polaires" (Grenier, 2009). La mythologie inclut ici le territoire, son climat et son écosystème, les peuples arctiques avec leurs traditions ancestrales et pratiques actuelles, et l'histoire des contacts avec les Européens.

$21 \mathrm{Au}$ nom de la découverte et de l'expérience de cette mythologie, le tourisme s'invite ainsi dans ces territoires nordiques et polaires. L'industrie touristique leur propose des activités de contact avec le milieu humain et l'habitat naturel, et des activités de dépassement de soi. L'illustration 6 recense les grandes catégories d'activités récréotouristiques offertes sur l'ensemble de l'Arctique circumpolaire. On y regroupe les activités de prélèvement (chasse et pêche), les activités d'observation (safaris motorisés ou en mode actif) et les activités culturelles et sportives. L'observation de la faune (la flore dans une moindre mesure) et du paysage constitue l'un des éléments de base de ce tourisme.

L'offre récréotouristique n'est pas répartie également sur l'ensemble du territoire arctique. Les pays nordiques, par exemple, dotés de réseaux routiers et ferroviaires modernes, ont ainsi développé depuis plusieurs décennies une offre touristique à la fois diversifiée et dynamique, allant du tourisme sur mesure au tourisme organisé (de masse $)^{4}$. En Amérique du Nord, la situation diverge du tout au tout, particulièrement dans le cas du Canada où le tourisme nordique et polaire n'a pas encore atteint son point de maturité (en référence au cycle de vie de la destination de Butler, 1980).

l'Arctique canadien, le climat, beaucoup plus sévère, freine considérablement le développement industriel et urbain. Le tourisme y est inévitablement moins présent. Outre les conditions difficiles du terrain et de son accès, plusieurs barrières additionnelles nuisent à son développement : une sacralisation (Jafari, 1988) inadéquate de ses attraits (à laquelle s'ajoute l'équilibre financier précaire entre, d'un côté, les coûts engendrés par la mise en tourisme et, de l'autre, ses retombées), des distances faramineuses entre les régions d'intérêt (faune, communautés, histoire), les coûts très élevés de la logistique et du transport (passagers et biens), le prix du pétrole, l'absence ou la défaillance des infrastructures d'hébergement et de transport local et la manque de formation des populations locales en matière de services touristiques. En Europe, où le territoire nordique est fortement développé, le tourisme se réalise principalement par voie terrestre. En Amérique du Nord, où il n'existe qu'une seule route franchissant le cercle polaire, l'avion et le navire deviennent les moyens de locomotion tout indiqués. 


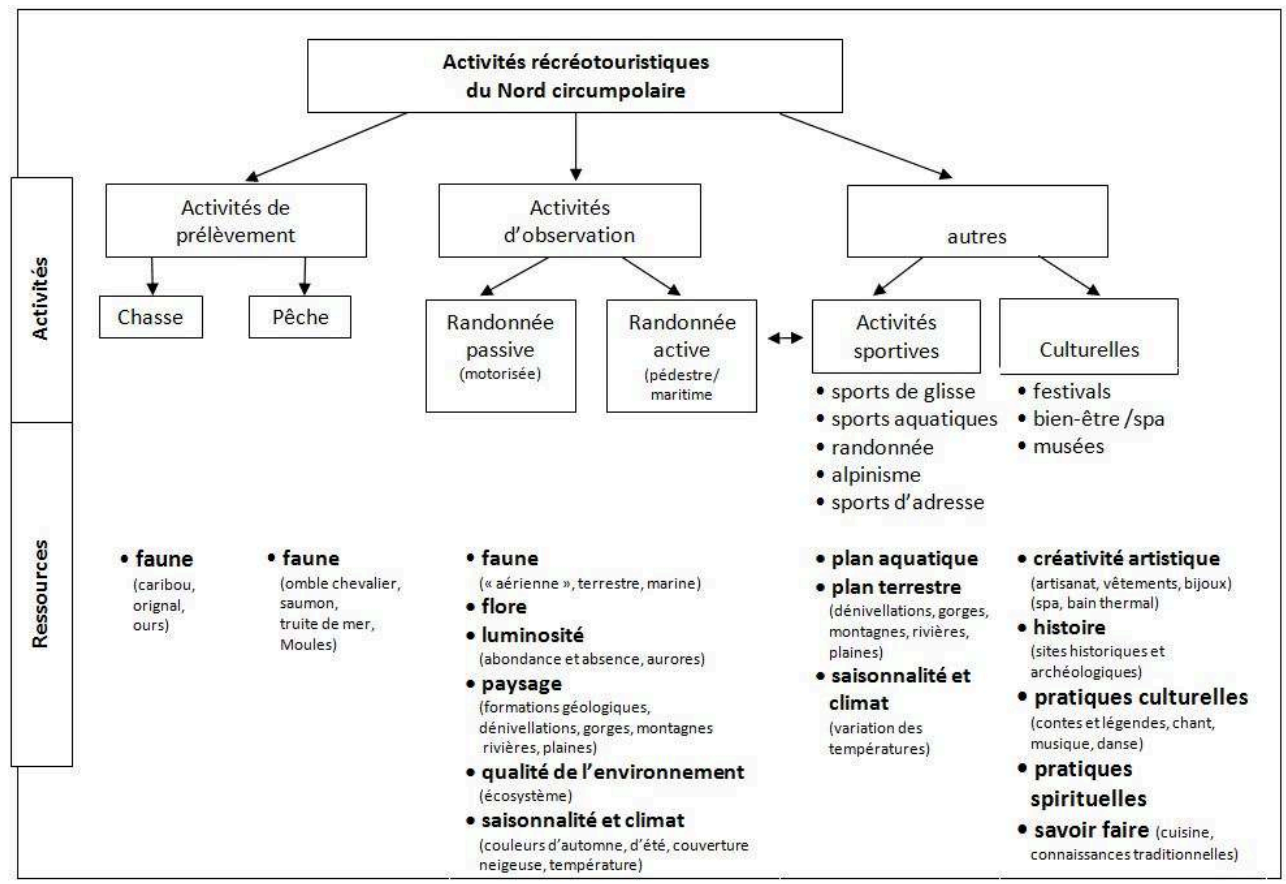

RECENSION DES ACTIVITÉS RÉCRÉOTOURISTIQUES DU NORD CIRCUMPOLAIRE

Source : Compilation de l'auteur à partir de ses observations et de l'offre de l'industrie.

\subsection{Croisières dans le passage du Nord-Ouest}

L'industrie de la croisière polaire a véritablement fait ses classes dans les eaux antarctiques. Elle y débarque à partir de 1957, de façon très sporadique. L'effondrement de l'économie soviétique, en 1991, libère la plus grande flotte de navires polaires. Récupérés par l'industrie de la croisière, ils propulsent depuis le voyage polaire par voie maritime de façon soutenue. Forte du succès qu'elle connaît en Antarctique, l'industrie met ensuite le cap sur d'autres destinations polaires dans l'hémisphère nord.

Par opposition aux croisières conventionnelles où le navire devient la destination, la croisière - le transport organisé d'un groupe (masse) de voyageurs par voie maritime, à des fins ludiques (Grenier, 2008) - devient une alternative plus " globale » aux yeux de nombreux touristes. Elle est prisée pour effectuer un premier voyage de "reconnaissance» pour se familiariser de façon très générale avec la diversité du territoire. Elle nécessite cependant beaucoup de temps (10 à 20 jours au moins) et ne consacre que quelques heures à chacun des milieux visités. Le navire peut aussi être un handicap lors du débarquement d'un nombre élevé de visiteurs (une centaine) dans des communautés isolées et encore relativement hermétiques. La prise en charge des visiteurs par des équipes sensibilisées et formées sur les réalités du Grand Nord, et par des accompagnateurs locaux, devient primordiale.

Tous types de navires confondus, ce sont les croisières (45 passagers et plus) et surtout les yachts de plaisance qui effectuent le plus de voyages dans le passage du Nord Ouest (voir Pharand, 2007: 31-33). Les yachts, aux circulations plus libres et imprévisibles que les navires de croisières, échappent à toute supervision. 
Le premier navire de croisière à s'aventurer dans le passage du Nord-Ouest fut le MS Explorer, en 1984. Il lui fallut 23 jours pour effectuer la traversée complète du passage, avec 98 passagers à bord, devenant du coup le $33^{\mathrm{e}}$ navire à réaliser l'exploit (Stewart et Draper, 2008 : 225). Comme ce fut le cas en Antarctique auparavant, l'industrie connaît des débuts lents. Seulement deux navires touristiques réussirent la traversée au cours des 4 années suivantes (Marsh et Staple, 1995). Entre 1992 et 2005, le nombre de navires de croisière dans le passage du Nord-Ouest s'accroit légèrement. Certains parviennent à compléter des traversées de l'Atlantique au Pacifique. D'autres proposent de nouveaux itinéraires vers la baie d'Hudson, la Terre de Baffin et même l'île d'Ellesmere (Stewart et Draper, $2008: 225)$.

À l'exception du Crystal Serenity aux étés 2017 et 2018, tous les navires de croisières employés à des fins touristiques ont jusqu'à présent été de classe polaire, c'est-à-dire disposant d'une proue renforcée (illustration 7). Avec environ trois navires de croisières dans les eaux de l'Arctique canadien par saison d'affaires, à raison de deux croisières consécutives en direction opposée (aller-retour), et avec en moyenne une centaine de passagers à leur bord, on peut très sommairement estimer à 600 le nombre de touristes de croisières qui visitent les hautes latitudes nord américaines, chaque été (août/septembre) (en excluant les yachts dont le nombre de passagers et les détails des débarquements au sol demeurent inconnus).

\section{Illustration 7}

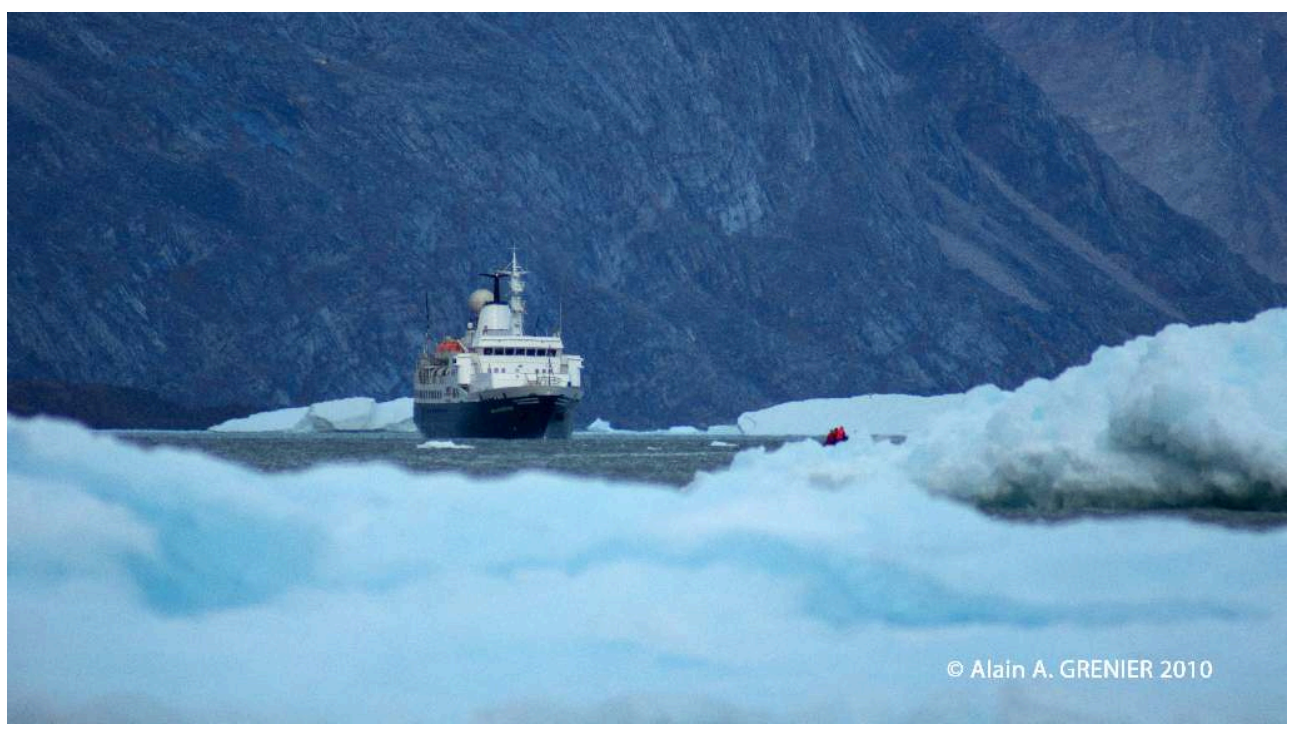

LE NAVIRE DE CLASSE POLAIRE (AVEC PROUE RENForCÉE) EST TOUT INDIQUÉ POUR NÉgocier SON PASSAgE dANS CES EAUX PARSEMÉES d'OBSTACLES de gLACE.

Source : Alain A. Grenier

Ces croisières proposent principalement :

- 1) des activités d'observation de paysages, de la faune et de la flore à partir du navire, d'embarcations pneumatiques (illustrations 8 et 9) ou de randonnées pédestres à l'occasion d'excursions au sol;

- 2) des visites de villages habités (en Arctique);

- - 3) des visites de lieux d'intérêt historique ou patrimonial (anciens postes de traite);

- - 4) des activités de sensibilisation ou d'éducation sur différentes thématiques touchant les milieux polaires (réalisées à bord du navire). 
Illustration 8

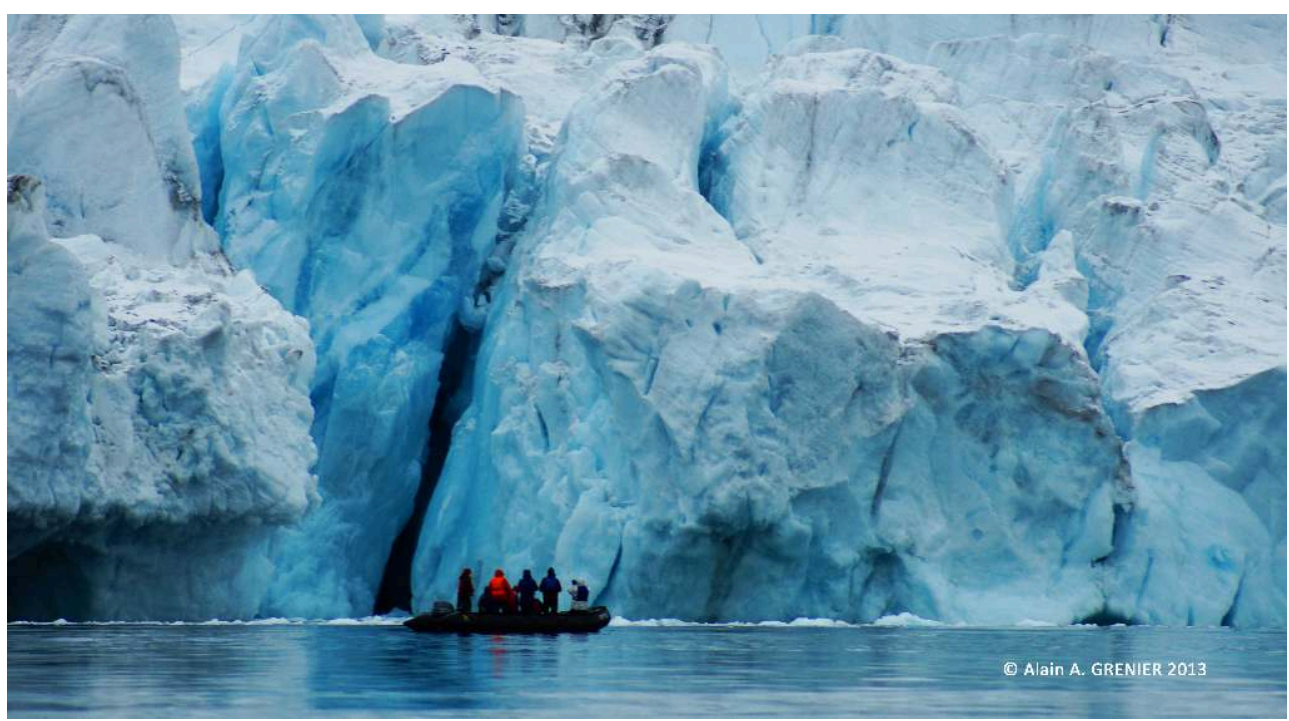

L'EXPLORATION DU MONDE POLAIRE, EN EMBARCATION PNEUMATIQUE, CONSTITUE UN POINT FORT DE L'EXPÉRIENCE DE LA CROISIÈRE POLAIRE.

Source : Alain A. Grenier

Illustration 9

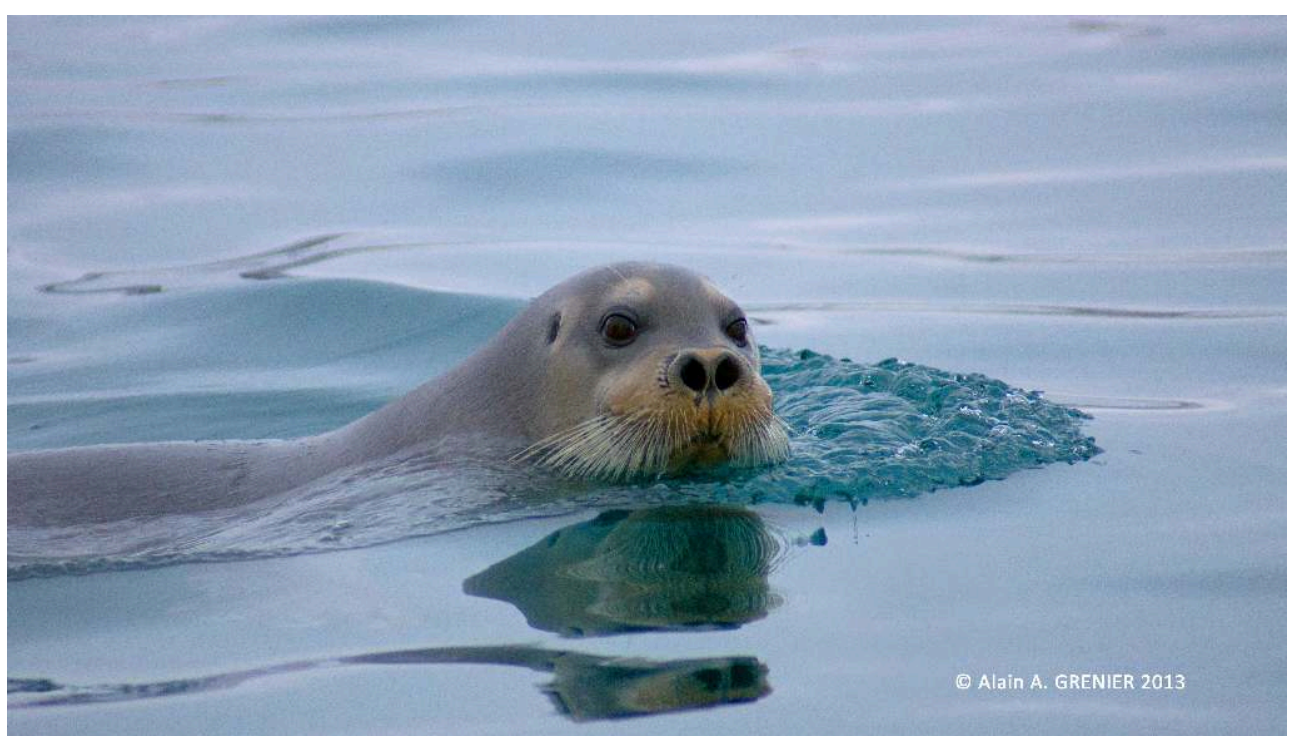

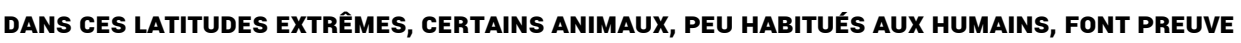
D'UNE gRANDE CURIOSITÉ, CE QUI LES REND VULNÉRABLES AUX VISITEURS MOINS BIEN INTENTIONNÉS.

Source : Alain A. Grenier

Ces activités répondaient, dans les années 1980 et 1990, aux besoins de groupes de touristes d'âge mur et âgés. L'âge moyen des croisiéristes polaire a désormais tendance à baisser, et les voyagistes ont commencé à offrir de nouvelles activités, un peu plus exigeantes sur le plan physique. Ces activités sont:

- - 5) de nature sportive (pratique du kayak de mer, de la course à pied, plongée sous-marine, activités de grimpe, etc.);

- 6) de nature bien-être (camping sous la tente en terre ferme, bains en eaux polaires, etc.). 
Ainsi, aux activités d'observation conventionnelles, de nature passive, s'ajoutent aujourd'hui des activités plus exigeantes sur le plan physique. Les pratiques sportives offrent une alternative stimulante aux touristes lorsque la faune brille par son absence. Sur le plan logistique, la distribution des attraits touristiques sur le Nunavut - un territoire de $2038722 \mathrm{~km}^{2}$, représente un défi organisationnel majeur pour les voyagistes. Les attraits ne sont pas répartis également sur le territoire. Leur appréciation nécessite ainsi des déplacements sur de vastes distances. L'arrivée de groupes de 100 à 200 passagers, dans le cas des navires de croisière, peut aussi constituer un casse-tête sur le plan logistique pour un territoire dépourvu d'infrastructures maritimes (port de mer, quais, débarcadères) et où les installations hôtelières ne sont pas toujours présentes ou de niveau. Les flots de visiteurs qui débarquent et explorent rues, plages, collines et même les cimetières de ces petites communautés dont le nombre d'habitants oscille de 1300 à 2000 personnes (outre la capitale, Iqaluit, qui compte près de 7000 habitants), peuvent engendrer un sentiment de dérangement pour les résidents, habitués à leur tranquillité (illustration 10).

Cela n'a pas empêché le Crystal Serenity de s'y aventurer en 2016 avec à son bord 1000 passagers et 600 membres d'équipage. Le plus grand navire à avoir tenté sa chance dans le passage du Nord-Ouest a complété une première traversée entre Seward (près d'Anchorage, Alaska) dont il était parti le 16 août, et la ville de New York jointe un mois plus tard. Malgré le prix (19 755\$ US/passager en classe économique, $120000 \$$ US / passager en première classe), tous les billets ont été vendus ! L'aventure fut répétée l'année suivante, à nouveau à guichet fermé.

Illustration 10

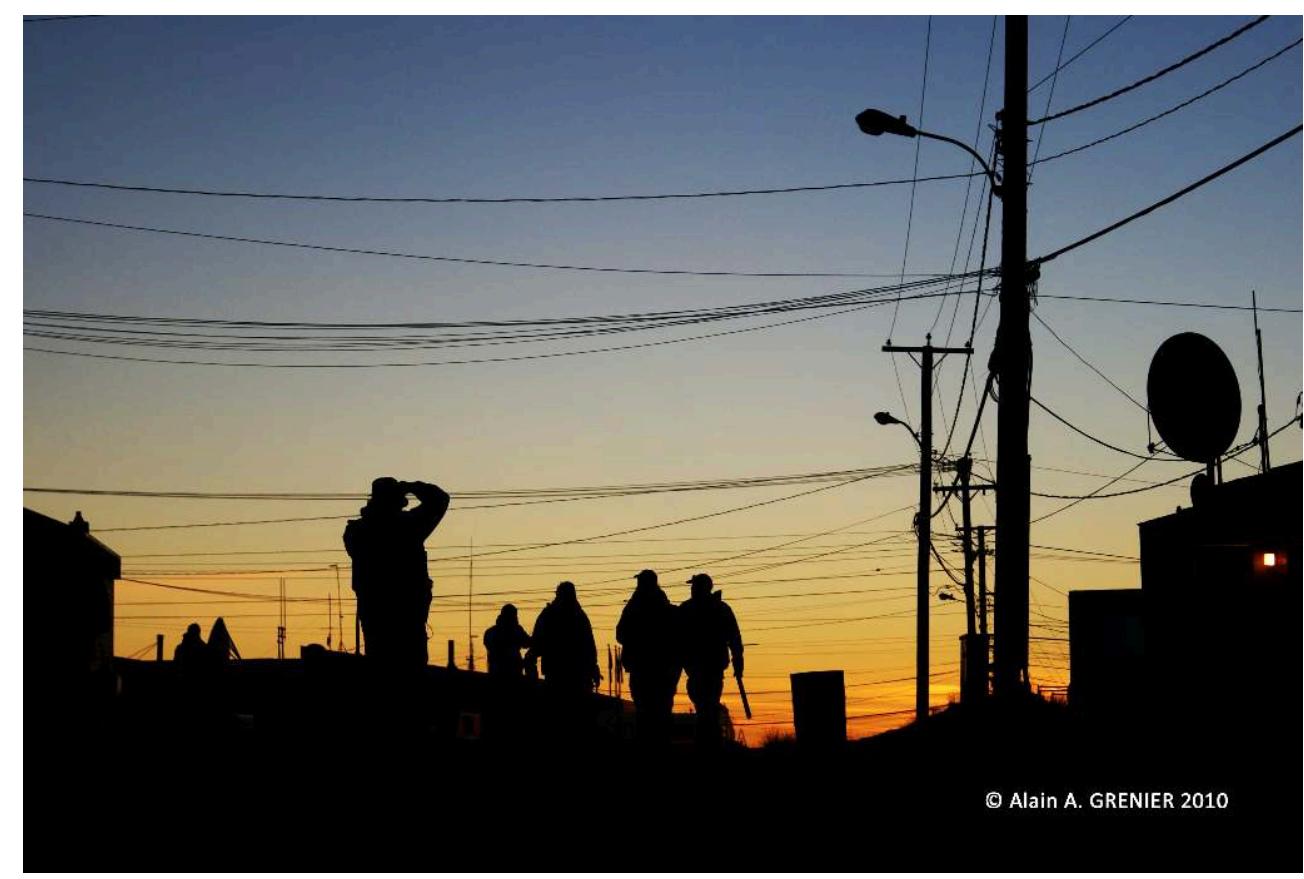

LORSQu'UNE CENTAINE DE TOURISTES débaRqueNt dANS UN VILLAgE INUIT, LES RÉSIDENTS PEUVENT SE SENTIR ENVAHIS.

SOURCE : ALAIN A. GRENIER, CAMBRIDgE BAY. 


\subsection{Portrait du tourisme de croisière au Nunavut}

Il est difficile de tracer un portrait du tourisme au Nunavut puisque le gouvernement territorial ne publie pas ses données de façon régulière. Seuls les voyagistes membres de l'Association of Arctic Expedition Cruise Operators (AECO) déclarent leurs passagers. On sait cependant qu'en 2015, le Nunavut a recensé 16750 visiteurs dont 1130 touristes «terrestres » et 2750 croisiéristes (AECO n'en recense que 422 pour la même année), auxquels s'ajoutaient 11550 touristes « d'affaires » (Nunavut Tourism, 2016:10). Il faut combiner les données de plusieurs sources pour arriver à dresser un portrait de l'industrie de la croisière au Nunavut (tableau 1). On remarque des différences importantes pour les mêmes catégories d'informations selon les sources. Le tableau permet néanmoins d'observer une croissance stable dans le nombre de croisiéristes, indiquant la vitalité de cette branche de l'industrie.

Tableau 1

\begin{tabular}{|c|c|c|c|c|c|c|c|c|c|c|c|c|c|c|c|}
\hline & 2005 & 2006 & 2007 & 2008 & 2009 & 2010 & 2011 & 2012 & 2013 & 2014 & 2015 & 2016 & 2017 & 2018 \\
\hline \multirow{6}{*}{ हैं } & $\begin{array}{l}\text { Nbre de passagers } \\
\text { (AECO, 2018, com pers.) }\end{array}$ & 179 & 214 & 230 & 124 & 264 & 239 & 124 & 272 & 485 & 317 & 422 & 1165 & 1199 & N.D. \\
\hline & $\begin{array}{r}\text { Nbre de passagers }{ }^{5} \\
\text { (NDEDT, 2018, } \\
\text { com.pers.) }\end{array}$ & N.D. & N.D. & N.D. & $2570^{5}$ & N.D. & 1398 & 1353 & 2153 & 3289 & 1905 & $\begin{array}{r}3364 \\
2750^{7}\end{array}$ & 4814 & 4972 & 4438 \\
\hline & $\begin{array}{r}\text { Nbre de voyagistes } \\
\text { (NDEDT, 2018, } \\
\text { com. pers) }\end{array}$ & N.D. & N.D. & N.D. & N.D. & N.D. & 6 & 4 & 6 & 6 & 7 & 8 & 8 & 9 & 10 \\
\hline & $\begin{array}{r}\text { Nbre de navires } \\
\text { (différents) } \\
\text { (NDEDT, 2018 } \\
\text { com.pers.) }\end{array}$ & N.D. & N.D. & N.D. & N.D. & N.D. & 7 & 5 & 6 & 8 & 8 & 11 & 11 & 12 & 11 \\
\hline & $\begin{array}{r}\text { Nbre de navires } \\
\text { (différents) } \\
\text { (Headland, 2018) }\end{array}$ & 2 & 2 & 2 & 1 & 2 & 3 & 1 & 2 & 5 & 1 & 2 & 3 & 3 & N.D. \\
\hline & $\begin{array}{r}\text { Nbre de croisières } \\
\text { (NDEDT, 2018, } \\
\text { com. pers.) }\end{array}$ & N.D. & N.D. & N.D. & N.D. & N.D. & 13 & 9 & 13 & 21 & 15 & 23 & 25 & 23 & 24 \\
\hline
\end{tabular}

Nombre de croisiéristes recensés dans le passage du Nord-Ouest (2005-2017) ${ }^{5}$

Entre 2006 et 2011, c'est la partie orientale, Qikiqtaaluk, qui reçoit le plus grand nombre de visiteurs, tous modes d'accès (avion, croisière) confondus (Gouv. Nunavut, sans date : 6). C'est normal, c'est là que sont concentrés les paysages les plus accidentés, et la faune marine. Cette région reçoit plus de $3 / 4$ des visiteurs tandis que les régions de l'ouest (Kittikmeot) et du centre (Kivaliq) reçoivent le reste, la région de l'ouest étant sensiblement favorisée (Gov. Nunavut, sans date : 6). À l'été 2018, les communautés les plus visitées ont été Pond Inlet (17 visites), Resolute, Gjoa Haven et Cambridge Bay (7 visites chacunes) et Grise Fjord (4 visites) (NDEDT, 2018, communications personnelles).

En général, les croisiéristes ont tendance à qualifier leur expérience de satisfaisante. Leurs frustrations viennent des journées entières passées en mer, surtout après la $2^{\mathrm{e}}$ journée de l'itinéraire. Les passagers veulent faire partie des privilégiés à traverser le passage du Nord-ouest. Mais cela demande de la patience. Un jour, par exemple, une excursion au Cap Félix, sur l'île du Roi-Guillaume, est reportée bien que la mer soit dépourvue de glace. Le chenal n'étant pas balisé, les cartes sont muettes sur la profondeur des eaux. L'équipage préfère ne pas s'y aventurer - un autre navire de croisière vient de s'échouer, à l'autre bout du passage, en voulant prendre un raccourci. Pour atteindre le site de l'excursion annoncée, l'équipage doit contourner l'île par un autre chemin, cartographié. Le détour fait perdre du temps, et ajoute à l'impatience. Quant on aperçoit tout à coup au loin un troupeau de bœufs musqués, le chef 
d'expédition choisit de ne pas s'arrêter, détour oblige. Même si l'on comprend que la nature ne soit pas un jardin zoologique, l'absence de faune à proximité du navire, engendre aussi la déception. "Venir jusqu'ici pour ne voir que des points noirs bouger", soupire une passagère, sarcastique.

Il est beaucoup plus difficile de satisfaire les gens dans l'Arctique qu'en Antarctique. «En Antarctique, explique une guide, nous disposons d'au moins 150 sites différents où nous pouvons amener nos visiteurs. Mais ici [en Arctique], tout ce que nous voulons montrer cherche à s'échapper ", souligne-t-elle. De plus, la destination est encore si nouvelle que l'équipage découvre souvent le territoire en même temps que les visiteurs. Les voyagistes se retrouvent ainsi entre l'arbre et l'écorce. Un voyagiste explique :

Si on n'énumère pas [dans les brochures] les animaux que l'on peut voir, les gens ne viendront pas. Si on leur dit qu'ils ne verront peut-être pas les animaux, ils ne viendront pas non plus. Les gens s'attendent à voir des ours polaires en masse. Ils ne savent pas [préalablement au voyage] que ce sont des animaux solitaires. Cela me dit que les gens ne font pas leurs devoirs.

Sans préparation adéquate, les attentes des touristes peuvent être irréalistes. "On sait bien que c'est du marketing", commente un touriste. "Nous ne sommes pas stupides. On sait qu'on ne verra pas grand-chose. Mais on se fait attraper et on espère obtenir les meilleures photos ». Pour augmenter leurs chances de rendre leurs clients heureux, les voyagistes doivent, après la visite des villages, dénicher les meilleurs sites "nature ", c'est-à-dire, pour l'observation de panoramas inhabituels (formations rocheuses et glaciers), et surtout des sites de prédilection pour l'observation de la faune, à proximité. Comme ils l'ont fait près de 30 ans plus tôt en Antarctique, les voyagistes font littéralement de l'exploration. Ils scrutent les chenaux et les baies, à la recherche d'expériences inusitées qu'ils pourront répéter. Il s'agit alors de choisir la bonne route. Or, le passage du Nord-Ouest est composé en réalité de 7 routes, chacune avec ses propres particularités.

\section{Les 7 routes du passage du Nord-Ouest}

Comme le passage du Nord-Ouest est en réalité une ouverture entre les îles de l'Archipel du Haut-Arctique et la rive septentrionale du continent nord-américain, il n'existe pas un mais 7 tracés possibles pour lier l'Atlantique au Pacifique, sans passer par le Pôle Nord (illustration 11). 
Illustration 11

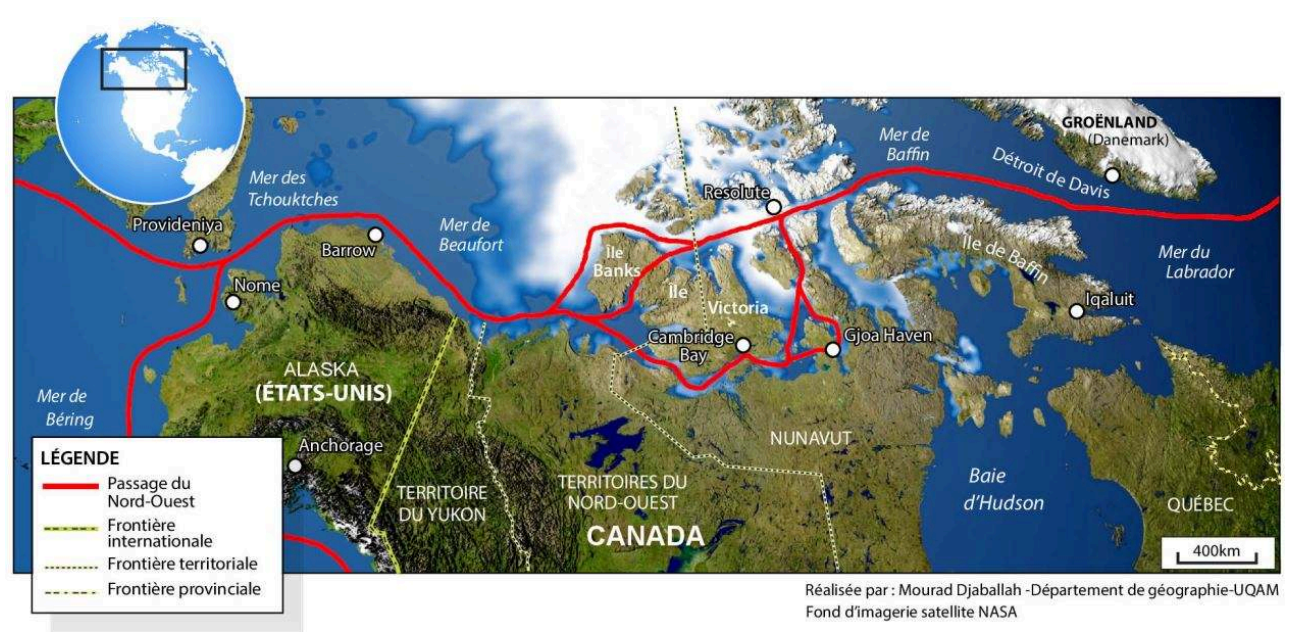

LES SEPT VOIES POTENTIELLES DU PASSAgE DU NORD-OUEST.

Source: Tracés de l'auteur sur carte de la NASA (domaine public).

En provenance d'Europe, le détroit de Lancaster, juste au nord de la Terre de Baffin, est la voie la plus appropriée à la traversée vers l'Asie. On s'engage alors dans les détroits de Peel, puis de Victoria et le golfe de la Reine-Maud pour ensuite rejoindre le Pacifique via les golfes du Couronnement et enfin d'Amundsen. C'est la route qui joint les communautés inuites les plus septentrionales. On peut aussi gagner le passage du NordOuest plus au sud via le détroit d'Hudson avant de traverser le bassin Foxe pour rejoindre le détroit de Fury et Hecla et de là le détroit de Bellot vers l'une des nombreuses sorties vers l'ouest. Outre la distance entre les deux océans, chacune des routes présente ses avantages mais aussi (et surtout) des inconvénients. Si l'intérêt des armateurs pour le passage du Nord-Ouest consiste dans l'économie de temps et d'argent pour le transit de leurs cargos par l'Arctique plutôt que par le très coûteux Canal de Panama, l'intérêt est fort différent du point de vue touristique. Chaque route revêt des degrés d'intérêt différents et mais aussi des défis logistiques. Le Tableau 2 propose un coup d'œil sur les caractéristiques de chacune des routes en fonction des intérêts touristiques et des défis de navigation. 


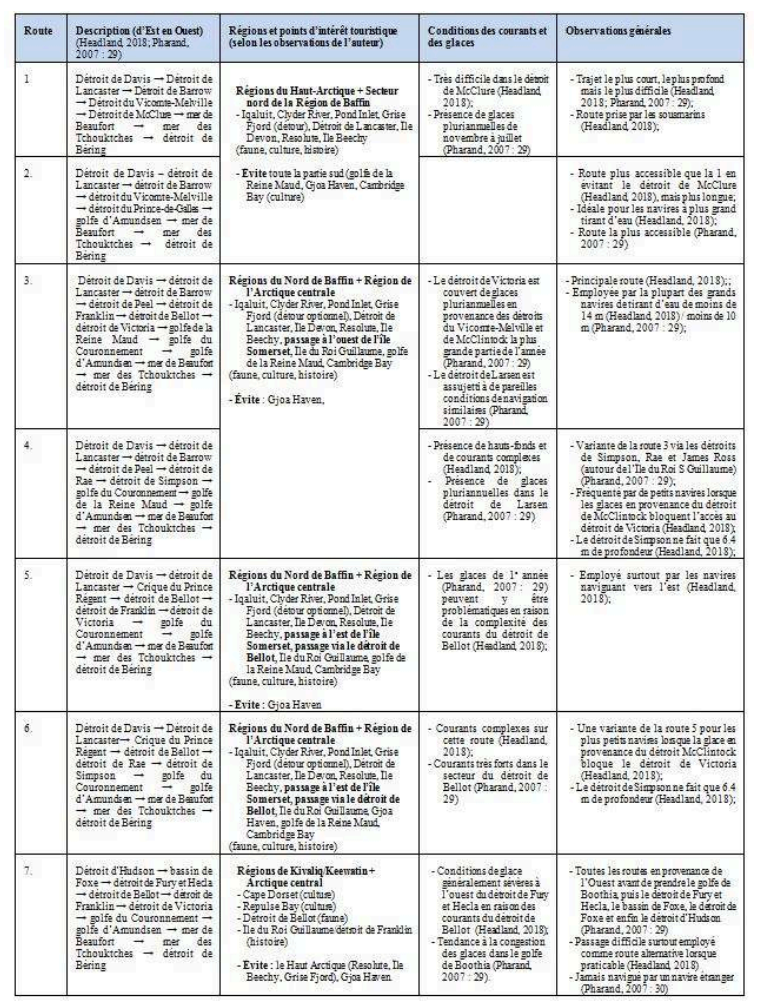

LES CARACTÉRISTIQUES DES 7 ROUTES DU PASSAgE DU NORD-OUEST EN FONCTION DES INTÉRÊTS TOURISTIQUES.

Source : observations de l'auteur pour la partie touristique, plus compilation à partir de Headland (2018) et Pharand (2007: 29).

41 Les croisières sont liées à un calendrier très strict d'itinéraires souvent organisés et vendus par différents voyagistes. Tout retard doit donc être comblé ou compensé à l'intérieur des dates d'embarquement et de débarquement annoncées aux passagers. Le choix de la route est alors dicté par la durée prévue pour la traversée, les courants marins, l'état des glaces et les conditions météorologiques. Les deux derniers facteurs sont irréguliers et imprévisibles. La traversée du passage du Nord-Ouest n'est ainsi jamais garantie (Hass et Howell, 2015).

42 Le navire à bord duquel j'ai pris place en 2013 a dû complètement changer son parcours en raison de glaces obstruant le golfe de la Reine-Maud et le détroit de Peel. Une semaine de navigation dans la partie ouest du passage fut remplacée par une exploration prolongée de l'Arctique de l'est. Il fallut transférer les touristes par vol nolisé de l'autre côté de l'Arctique, pour réaliser le nouvel itinéraire. La réaction des passagers, informés la veille du départ, fut contenue. Certains exprimaient leur déception à ne pas pouvoir « revendiquer » avoir fait la traversée complète du passage. D'autres trouvaient leur compte dans le fait que le voyagiste leur proposait en remplacement d'explorer davantage les latitudes les plus septentrionales du détroit de Smith. On reconnait ici un trait des touristes de croisières, celui du conquérant (Grenier, 2004: 400), dont la motivation première est d'atteindre des points géographiques auxquels peu d'humains ont accès.

Le mouvement des glaces force ainsi souvent les équipages à improviser un parcours via plusieurs segments de routes du passage du Nord-Ouest. En général, les routes 6 et 4 
sont les plus employées par les navires de croisières. La route 6 permet notamment de joindre un maximum de points d'intérêt. En effet les voyagistes doivent parallèlement tenter de garder dans leur parcours des sites prisés de l'Arctique canadien. À ce niveau, le fait que le tourisme y soit encore dans sa phase de développement, signifie que peu de sites ont été « sacralisés » (voir MacCannell, 1976, r. 1989) et popularisés dans les outils de promotions, comme c'est le cas désormais en Antarctique. L'île Beechy, avec les sépultures de l'expédition Franklin, fait exception. L'île, relativement accessible, s’impose comme site de visite sur la majorité des itinéraires (illustration 12).

\section{Illustration 12}

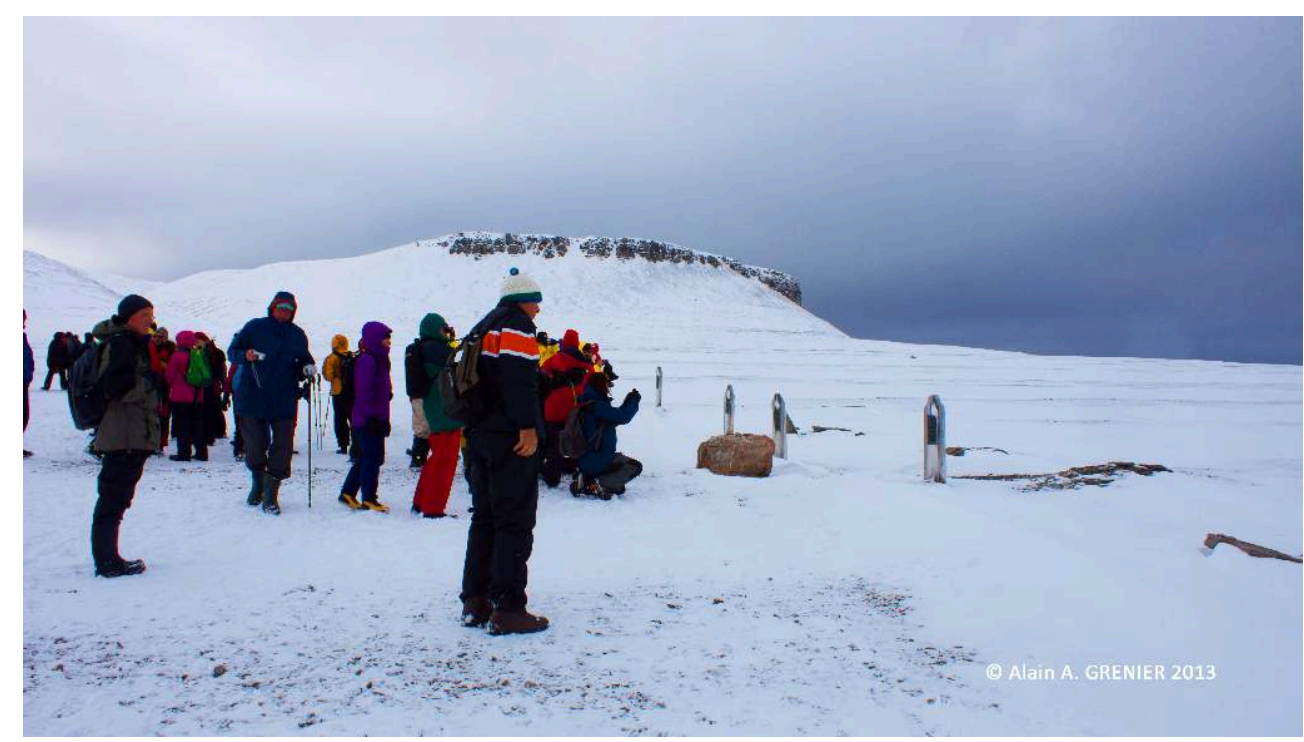

L'ÎLE BEECHY EST LE LIEU DU HAUT ARCTIQUE LE PLUS VISITÉ. LES TOURISTES S'ATTROUPENT AUTOUR DES PIERRES TOMBALES DES PREMIERS MARINS DE L'EXPÉdITION FRANKLIN À AVOIR TROUVÉ LA MORT.

Source : Alain A. Grenier

Bien que les visites de communautés inuites ne figurent généralement pas parmi les attentes les plus élevées des visiteurs (aucune attente recensée auprès d'eux lors de l'étude de 1999; à peine $6 \%$ et $4 \%$ dans les études de 2010 et 2013), plus de $25 \%$ des passagers déclarent ensuite qu'elles ont été parmi les moments les plus mémorables du séjour. On peut interpréter ce revirement par la démystification de l'Inuit, rendue possible par le contact, si bref soit-il, entre les visiteurs et une communauté. Cela passe généralement par un arrêt formel dans le gymnase de l'école ou de son centre communautaire pour une démonstration de pratiques traditionnelles (jeux arctiques, "défilé de mode » de vêtements traditionnels en peaux d'animaux, chants de gorge, etc.) (Illustration 13). 
Illustration 13

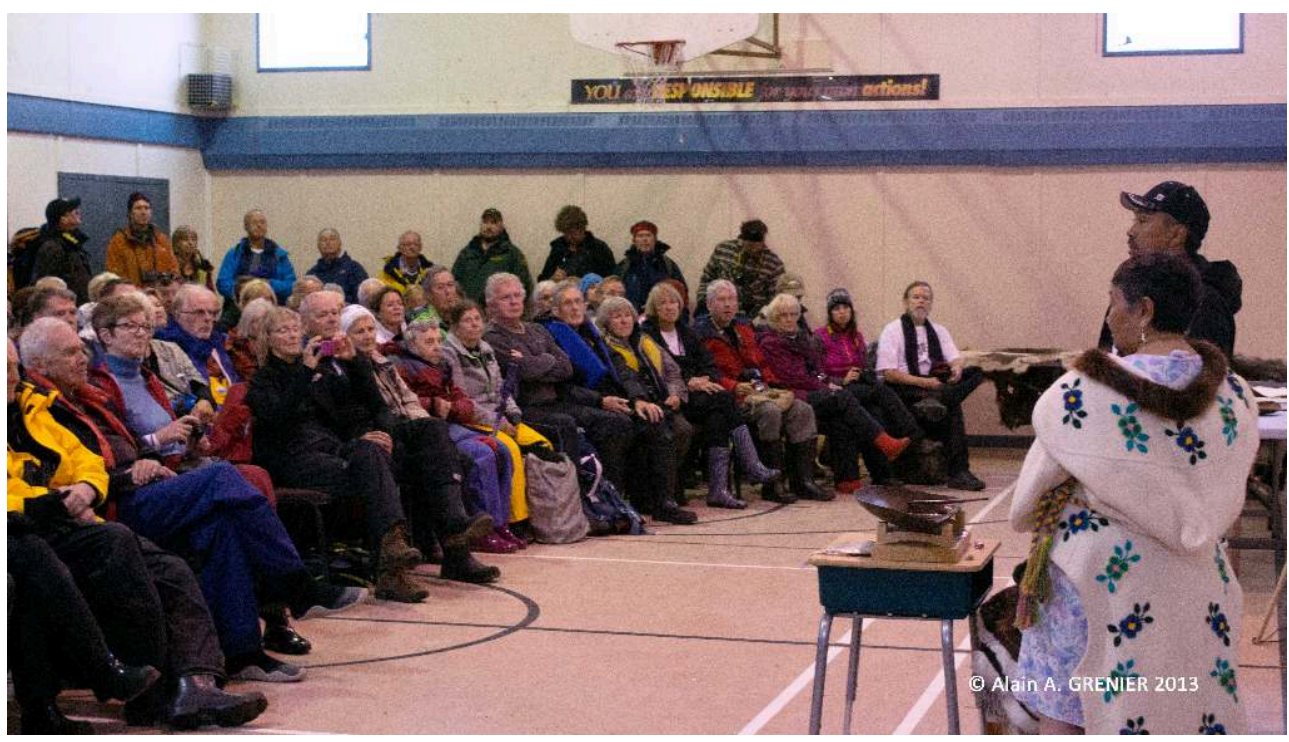

UN gROUPE DE CROISIÉRISTES PARTICIPE À UNE DÉMONSTRATION DE SAVOIR-FAIRE DE LA POPULATION INUITE DE GRISE FJORD.

Photo : Alain A. Grenier

mal informés quant à la vie de ces peuples arctiques et leur transformation depuis les premiers contacts avec les Européens. Arrivés dans ces villages avec comme seules images celle de l'Inuit urbain souvent en difficulté dans le Sud, ou celle des productions artistiques (sculpture principalement), les croisiéristes ont l'occasion d'ouvrir leur esprit pour corriger leur perception de cette population. Le contact entre les visiteurs et les visités n'est pas toujours facile. Des incidents de nature socioculturelle notamment des insultes proférées par les visiteurs - sont parfois rapportés par les Inuits. Pour autant, dans l'ensemble les touristes repartent avec davantage de compassion pour cette population et sa capacité d'adaptation à cet environnement. Quoique les touristes évaluent favorablement les paysages pour leur grande beauté, l'environnement des Inuits demeure néanmoins très hostile, d'où ce respect accru des touristes à leur égard. En revanche, le taux d'appréciation des visiteurs pour la faune fond au soleil, elle qui se fait discrète et rarement aussi accessible que ne le laissent croire les brochures, abondamment illustrées de photographies.

Si le tourisme est généralement considéré comme un outil de développement économique, ce n'est pas vraiment le cas du tourisme de croisières polaires. En 2015, les croisiéristes représentaient $16 \%$ du nombre total de visiteurs du Nunavut, une augmentation de $46 \%$ depuis 2011 (Nunavut Tourism, 2016: 10). Pourtant, ils n'ont laissé que $5 \%$ des 38 millions de dollars (CAN) de revenus engendrés par le tourisme. Les croisiéristes de l'Arctique ont la réputation de laisser moins dans les communautés que les autres types de touristes (Gov. Nunavut, sans date : 18). Il faut dire que le touriste paie en moyenne $17000 \$$ (CAN) pour sa croisière (Nunavut tourism, 2016: 11). Les billets de croisière ne rapportent pas au territoire visité, contrairement aux billets d'avion puisque les Inuits sont les principaux actionnaires des deux grandes compagnies aériennes à desservir leur territoire. Considérant que les croisiéristes se déplacent avec leur propre hôtel, leur restaurant et leurs guides, il ne reste que les souvenirs et les activités au sol, lors des excursions, pour dépenser un peu dans les 
villages. À défaut d'activités payantes lors des visites de villages, les touristes n'y laissent que quelques dollars pour l'achat de souvenirs.

Des artistes inuits approchent souvent les touristes sur la rue pour leur vendre des sculptures. Comme les transactions se font dans l'atmosphère informelle de la rue (et non en boutique), le touriste négocie. Il n'est pas rare de voir des situations où une sculpture d'inukshuk d'environ $10 \mathrm{~cm}$ de hauteur ne trouve pas preneur à 40 \$ (CAN) (trois fois moins cher qu'en boutique, dans les villes du Sud) pour constater, de retour sur le navire, que certains touristes arborent leurs " prises " négociées à moitié ou au quart du prix. Pendant ce temps, le gouvernement territorial souhaite encourager la promotion des artistes locaux afin d'amener les croisiéristes à passer davantage de temps dans les villages (Nunavut tourism, $2016: 11$ ).

Quant aux voyagistes, ils aimeraient proposer davantage d'itinéraires à leurs clients mais ils se plaignent du degré élevé de la bureaucratie canadienne en comparaison avec d'autres milieux d'accueil jugés plus réceptifs comme le Groenland (Nunavut Tourism, 2016 : 11). Mais il y a plus problématique encore : la question de la sécurité des navires, de leurs équipages et de leurs passagers.

\section{Dangers de la navigation dans le passage du Nord- Ouest}

De tous les risques auxquels s'exposent les navires et leurs passagers, en territoires polaires, la présence de glaces et les hauts fonds sont les plus importants. Les incidents impliquant des navires de croisière dans les régions polaires ont longtemps échappé au grand public en l'absence de médias pour les rapporter. Dans la mesure où l'équipage arrivait à se tirer d'affaire sans aide extérieure, et sans contrecoup, l'incident passait « sous le radar».

50 L'arrivée de l'Internet et des téléphones intelligents a changé les choses, en donnant aux touristes la chance de rapporter eux-mêmes ce qu'ils voient, comme ce fut le cas lors du naufrage du MS Explorer le 23 novembre 2007, en Antarctique. L'Explorer s'était aventuré, au plus profond de la nuit, dans une zone glacée que l'équipage croyait être une glace de première année (peu épaisse et malléable). L'enquête a plutôt démontré qu'il s'agissait de glace de seconde année, beaucoup plus résistante. Or, le navigateur en charge au moment du contact fatal, n'était pas familier avec les conditions de navigation de l'Antarctique (Maritime Executive, 2009). Les 154 passagers et membres d'équipage de l'Explorer ont trouvé refuge dans les embarcations de sauvetage ouvertes et sans protection face aux éléments, tels le vent et le froid, ce qui, en milieu polaire, diminue les chances de survie. Ils n'ont été secourus que le lendemain par d'autres navires de croisière, qui avaient été détournés de leurs itinéraires respectifs. Deux heures après qu'ils ont été secourus, des vents violents s'abattaient sur la zone du naufrage.

51 L'Arctique n'est pas en reste. Jusqu'à présent, les accidents impliquant des navires de croisière dans le passage du Nord-Ouest, ont été moins sévères que ceux rapportés dans l'Antarctique (voir Tableau 3). Tous ont été causés par la présence de hauts fonds - dont l'existence de certains était connue. Cela n'aura pas suffit à empêcher les navires de s'y échouer. Tous ont nécessité l'évacuation des passagers en raison de la position précaire du navire ou de la perforation de sa coque. 
Tableau 3

\begin{tabular}{|c|c|c|}
\hline Année et lieu & $\begin{array}{l}\text { Navire et } \\
\text { voyagiste }\end{array}$ & Nature de l'incident et repercussions \\
\hline $\begin{array}{l}2018 \text { ( } 24 \text { août) } \\
\text { Kugaaruk, } \\
\text { Nunavut }\end{array}$ & $\begin{array}{l}\text { Akademik } \\
\text { Ioffe }\end{array}$ & $\begin{array}{l}\text { - Échouage } \\
\text { - Évacuation et transfert des } 162 \text { passagers et membres } \\
\text { d'équipage vers un autre navire- le Akademik } \\
\text { Sergey Vavilov; } \\
\text { - Entrée d'eau à bord du navire; } \\
\text { - Aucune indication d'impact environnemental; } \\
\text { - Assistance au navire : deux brise-glaces canadiens } \\
\text { (Pierre Radisson et Amundsen), deux avions } \\
\text { Hercules, deux hélicoptères de type Cormorant. } \\
\text { (CBC News, 2018) }\end{array}$ \\
\hline $\begin{array}{l}2010 \\
\text { Golfe du } \\
\text { Couronnement, } \\
\text { près de } \\
\text { Kugluktuk } \\
\text { (Nunavut) }\end{array}$ & $\begin{array}{l}\text { MV Clipper } \\
\text { Adventurer }\end{array}$ & $\begin{array}{l}\text { - Échouage sur une plate-forme rocheuse } \\
\text { - Évacuation des } 128 \text { passagers et } 69 \text { membres } \\
\text { d'équipage; } \\
\text { - Déversement dans la mer de carburant et de boues } \\
\text { (eaux noires) en provenance de } 13 \text { réservoirs } \\
\text { perforés; } \\
\text { - Assistance au navire : le brise glace canadien } \\
\text { Amundsen et quatre remorqueurs. (Hinchey, 2017) }\end{array}$ \\
\hline $\begin{array}{l}1996 \\
\text { Près de } \\
\text { Cambridge Bay } \\
\text { (Nunavut) }\end{array}$ & Hanseatic & $\begin{array}{l}\text { - Échouage } \\
\text { - Évacuation des } 150 \text { passagers et membres } \\
\text { d'équipage (Kenney, 2006:181) }\end{array}$ \\
\hline
\end{tabular}

LISTE d'INCIDENTS DE NAVIgATION IMPLIQUANT DES NAVIRES DE CROISIÈRE DANS LE PASSAgE DU NORD-OUEST.

Source : Compilation de l'auteur

Sur les mers glacées, nul navire, même de classe polaire, n'est à l'abri d'un accident. Leurs conséquences peuvent être graves, tant sur le plan humain (blessures, hypothermie, risque de mort) que sur celui de la logistique (organisation des secours, renflouage et remorquage des navires). Sur le plan environnemental, ils nécessitent la récupération des matières polluantes et le secours à la faune, à la flore. Toutes ces opérations engendrent des coûts et engagent la responsabilité des acteurs qui les ont causés puis subis.

Les cas du naufrage de l'Explorer, en Antarctique, et la médiatisation de l'échouage du MV Clipper Adventurer, en 2010, ont permis de mettre en lumière certains de ces aspects. Ces accidents rappellent l'importance de l'expérience des navigateurs et de la formation des équipages pour les eaux polaires (Maritime Executive, 2009). Ils confirment aussi un important problème de sécurité dans le choix des routes navigables. À ce sujet, il est (re)connu que les cartes de navigation du passage du NordOuest datent du milieu du XXe siècle et sont souvent erronées (Geiger et Mitchell, 2015 : 23). Plusieurs intervenants ou spécialistes ont déploré cette situation dans la presse (Cole, 2018; CBC News, 2008). En dépit des avancées technologiques, notamment en matière d'exploration et de cartographie sous-marine, la connaissance du territoire arctique canadien demeure largement incomplète. Un voyagiste explique que "seulement $10 \%$ de la partie canadienne de l'Océan arctique a été cartographiée avec des données vieilles de 50 à 60 ans réalisées avec des équipements peu fiables ", ce que confirment Geiger et Mitchell (2015: 10). Pour sa part, la Garde côtière estime à $27.6 \%$ 
le territoire de navigation couvert par des activités hydrographiques à la fois modernes et adéquates (GCC. 2018a, communications personnelles). La situation n'est pas unique au passage du Nord-Ouest. La Garde côtière souligne que la situation est semblable pour la navigation autour des îles Haïda Gwaii (anciennement îles de la Reine-Charlotte, sur la côte ouest de la Colombie-Britannique, de même qu'autour de l'île de Terre-Neuve (GCC, 2018b). Même son de cloche du côté de l'Alaska, aux États-Unis, où seulement 4.1 \% des côtes arctiques de l'État seraient cartographiées selon les normes actuelles (Cole, 2018). Chaque été, le gouvernement canadien déploie des navires pour cartographier des sections du passage du Nord-Ouest. "C'est un travail de minutie qui nécessite beaucoup de fonds publics", explique un voyagiste. Cinq années peuvent s'écouler entre la collecte des données, leur analyse et leur retranscription sur des cartes. « Au rythme où le travail se fait, commente le voyagiste, couvrir l'ensemble des routes pourrait nécessiter 300 ans ». Or, le mandat premier de la Garde côtière n'est pas la cartographie mais plutôt l'escorte des navires de ravitaillement, y compris des pétroliers qui desservent les villages de l'Arctique chaque été.

54 À défaut de voir l'État canadien (Service hydrographique du Canada) se consacrer entièrement à la cartographie des fonds marins, d'autres prennent l'initiative. Les recherches menées ces dernières années pour retrouver les épaves de l'expédition Franklin ont permis de cartographier les fonds marins de plusieurs secteurs du passage du Nord-Ouest. Ainsi, le détroit d'Alexandra, à l'ouest de l'île du Roi Guillaume, disposera dorénavant de cartes à jour, permettant sa navigation en toute sécurité. Les navires pourront du coup épargner 6 à 7 heures de navigation et de carburant, entre Cambridge Bay et Repulse Bay, sans devoir passer par Gjoa Haven lorsque le détroit de Victoria est glacé (Davison, 2016).

La question du secours aux navires et à leurs passagers est également cruciale. Le Canada dispose de très peu de moyens pour aider un navire en détresse à ces latitudes. Sa flotte de brise-glaces vieillissante nécessite d'être renouvelée. De plus, les Forces armées et la Garde côtière voient leurs budgets constamment réduits, affaiblissant du coup les capacités du Canada à porter secours à tout navire en danger, une condition sine qua non pour qui veut assumer la souveraineté de son territoire (Huebert, 2011 : 392, 394). Les autorités canadiennes ont plutôt choisi d'investir dans la surveillance terrestre en créant un corps de Rangers attachés à la Réserve des forces armées canadiennes (FAC). Ces rangers constituent des «forces mobiles autosuffisantes [...] dotées d'équipement léger pour appuyer les opérations des FAC en matière de sécurité nationale et de sécurité publique " dans les régions éloignées, côtières et isolées du Canada (Armée canadienne, non daté). Cette force terrestre pourrait-elle porter secours à un navire en détresse alors qu'il transporte des centaines de passagers ?

En Arctique, le temps nécessaire pour atteindre le lieu d'un accident maritime varie en fonction de la position du navire en détresse, de la localisation de navires de la Garde côtière (ou tout autre navire), de la direction des courants marins, de la présence de glace (à contourner), de la puissance des navires de secours et de leur autonomie énergétique. Les premiers intervenants risquent ainsi d'arriver par les airs. Or, les bases les plus proches de l'Aviation royale canadienne sont toutes situées hors de la zone arctique, dans les provinces du sud (Cold Lake en Alberta, Bagotville au Québec et Goose Bay au Labrador). Le Canada dispose d'une unité spécialisée en recherche et sauvetage. "Dans le cas où une intervention requerrait de gérer un grand nombre de personnes, affirme un conseiller en communication de la Garde côtière (GCC, 2018a, 
communications personnelles), une trousse en cas de catastrophe aérienne (CATAIR) peut être utilisée. Cette trousse est conservée à la base des forces canadiennes de Trenton, en Ontario, et peut être transportée dans deux aéronefs Hercules et larguée par parachute avec des techniciens de recherche et sauvetage sur le site d'un écrasement d'aéronef ou sur un site d'évacuation terrestre dans le cas de l'échouement d'un navire. La trousse est composée de tentes, de chaufferettes, de nourriture, d'eau, de couvertures, de fournitures médicales, etc. »

La base de Trenton, ainsi chargée des opérations de sauvetage pour la plus vaste partie du Grand Nord canadien, est située dans le sud de l'Ontario. Elle est à toutes fins pratiques plus près de Quito (Équateur) que de la base d'Alert, au Nunavut (CBC News, 2010). "Les aéronefs Hercules peuvent décoller en 12 heures; le temps de vol doit être ajouté à ce chiffre", souligne notre source à la Garde côtière (GCC, 2018a, communications personnelles) qui ajoute qu'elle dispose néanmoins de 15 unités auxiliaires réparties dans l'Arctique et qui peuvent lancer une intervention locale. Mais le temps nécessaire pour se rendre sur les lieux dépend de plusieurs facteurs; chaque cas étant unique (GCC, 2018a, communications personnelles).

Néanmoins, faut-il rappeler le cauchemar des survivants de l'accident d'un appareil Hercules C-130 des forces armées canadiennes, le 30 octobre 1991, sur l'île d'Ellesmere, qui ont dû patienter 32 heures par des températures de $-20^{\circ} \mathrm{C}$ à $-30^{\circ} \mathrm{C}$ avant d'être secourus (Thomas, 2015). On reconnaît ici le syndrome canadien des services publics disposés à proximité des grands bassins de population, dans le sud, alors que le pays est géographiquement dominé par un territoire nordique, voire polaire. Cette situation fait dire à une source gouvernementale, qui requiert l'anonymat, qu'elle comprend que les touristes veulent atteindre le passage du Nord-Ouest mais qu'elles doivent considérer les risques, comme pour ceux qui font de l'alpinisme.

Au-delà des impacts humains, les impacts écologiques ne doivent pas être minimisés. Toute ouverture dans la coque ou la quille d'un navire peut entrainer le déversement en mer de matériau pouvant compromettre l'intégrité de l'écosystème. Au Canada, les populations inuites dépendent encore largement de la pêche (et de la chasse) pour subvenir à leurs besoins alimentaires et maintenir leurs pratiques culturelles traditionnelles. Le bruit des navires perturbe certaines espèces marines sensibles à la pollution sonore (bélugas et narvals, notamment) et dont se nourrissent les Inuits. De plus, les coques des navires transportent avec elles des organismes parasites qui peuvent trouver, dans ces eaux, de nouveaux territoires à coloniser, délogeant du coup des espèces locales moins résilientes (Chan et al., 2012: 7). Les navires émettent aussi des particules polluantes, dont le noir de carbone (produit par la combustion incomplète d'hydrocarbures) et des gaz à effets de serre.

\section{Conclusion}

Dans le contexte des changements climatiques, le passage du Nord-Ouest ravive les rêves d'une voie navigable pour différents groupes d'intérêts (armateurs et États) qui y voient des opportunités de développement économique et d'affirmation politique. Jusqu'à présent, les glaces, encore très présentes même si instables, freinent l'action de ces groupes d'intérêts. C'est plutôt l'industrie du tourisme, toujours assoiffée d'originalité, qui y déploie depuis trois décennies, ses navires de croisière.

61 Ce tourisme repose sur l'exploitation des ressources naturelles. À cela s'ajoutent quelques attraits culturels (coutumes et pratiques des populations autochtones) et 
historiques de ces régions. Plus que tout, c'est son rapport avec l'aventure et par la prise de risques très contrôlés qu'il s'octroie l'accès à des territoires souvent peu fréquentés, voire rarement explorés par les humains.

L'industrie de la croisière polaire se fait ainsi l'instigatrice de l'exploration et de la documentation de cette route maritime qu'est le passage du Nord-Ouest (illustration 14). Par ses itinéraires entrecroisés parmi les 7 routes qui composent ensemble le passage du Nord-Ouest, elle explore, comme l'éclaireur, les meilleurs lieux d'escale pour l'observation de la faune et du paysage.

Illustration 14

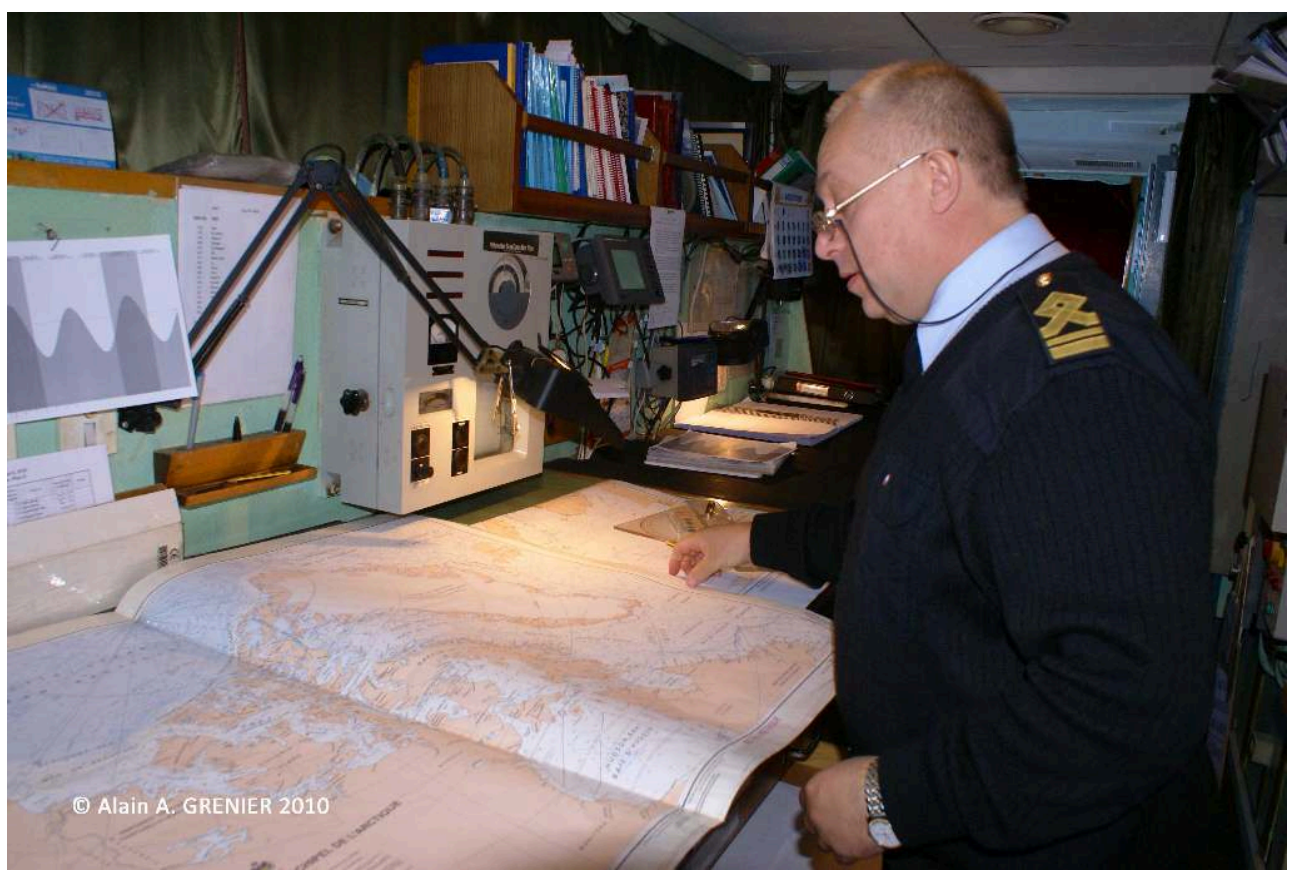

PAR L'ENTREMISE de SES ÉquiPAgES, L'INDUSTRIE de LA CROISIÈRe POLAIRE COLLECTE BEAUCOUP D'INFORMATIONS SUR LE TERRITOIRE ARCTIQUE ET SON PASSAgE DU NORD-OUEST, QUI SERVIRONT UN JOUR AUX PÉTROLIERS ET AUTRES NAVIRES CARgOS QUI ATTENDENT, PATIEMMENT, L'OUVERTURE SÉCURISÉE DE LA ROUTE.

Source : Alain A. Grenier

À l'instar des autres formes de voyage de détente, le tourisme polaire soulève aussi des réserves quant à ses impacts sur les cultures hôtes et leur environnement (Mason, 2010 : 151). Ce questionnement résulte des changements radicaux, souvent irréversibles que le tourisme entraine dans son sillon sans toujours en connaître les revers ou savoir comment les contenir (illustration 15). Des malentendus culturels entre Inuits et visiteurs lors de croisières polaires ont été rapportés, malgré les précautions prises par les voyagistes comme les organisateurs locaux pour minimiser les incompréhensions. Ces attentions sont d'autant plus importantes que les Inuits, après être passés d'une culture vivrière à l'ère moderne en moins d'un demi-siècle, sont aujourd'hui assujettis à une nouvelle phase de décloisonnement. Les changements climatiques amènent la possibilité que le passage du Nord-Ouest, jadis maudit par les explorateurs qu'il a décimés, soit bientôt libéré des glaces et ouvert à la navigation commerciale. Le tourisme, première grande manifestation de la mondialisation, stimule plus que tout autre acteur ce décloisonnement de l'Arctique. 


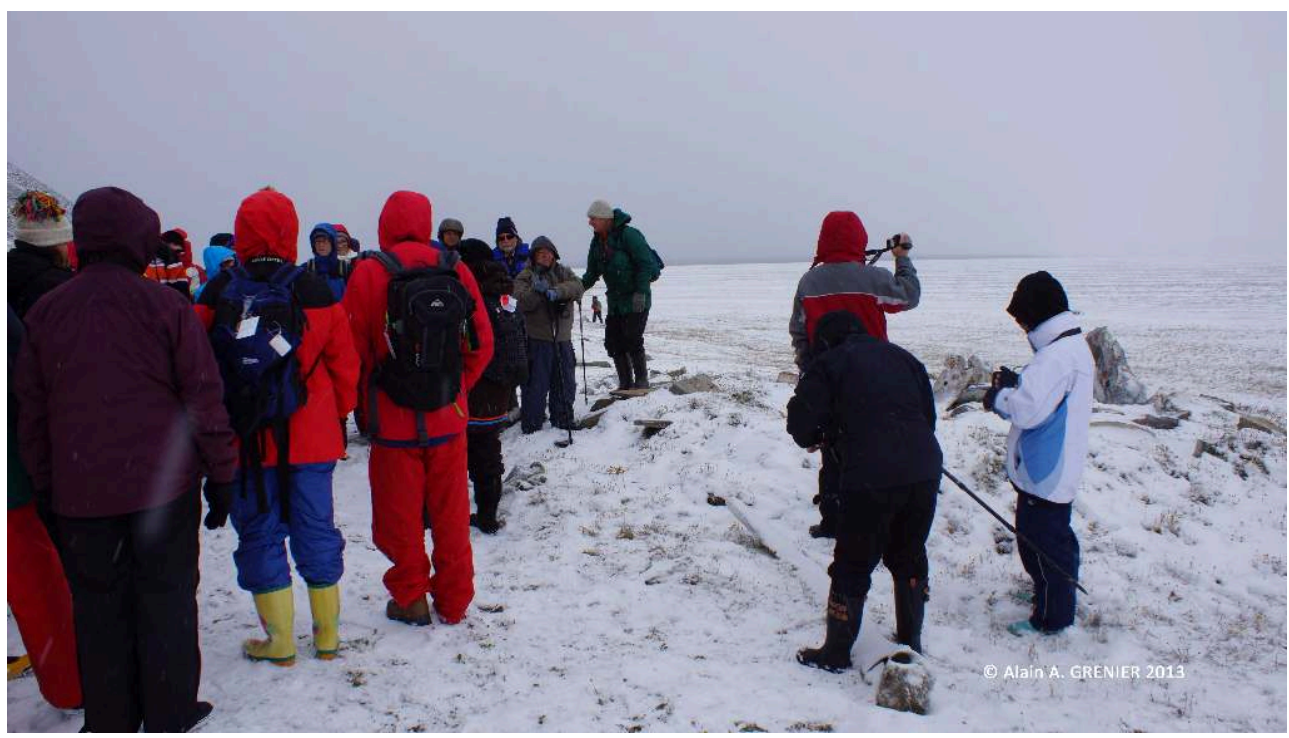

DES TOURISTES, FRAICHEMENT DÉBARQUÉS SUR UN SITE D'INTÉRÊT, PIÉTINENT DES ARTEFACTS INUITS (UNE HABITATION) SANS EN ÊTRE CONSCIENTS.

Source : Alain A. Grenier

Le passage controversé du Crystal Serenity et de ses 1700 passagers annonce, une nouvelle vague de touristes, encore plus assoiffés de sensations fortes. C'est ce que laisse présager le nouveau type de navire qui se construit dans les chantiers navals. Un voyagiste fait déjà la promotion de son nouveau navire, le Crystal Endeavor, présenté comme un «méga-yacht de classe polaire » (Crystal, 2016; traduction libre de l'auteur) (Illustration 16). Quoique de capacité beaucoup plus modeste (200 passagers et 200 membres d'équipage, ce qui est néanmoins considérable pour l'Arctique), ce navire construit spécialement pour les croisières en eaux polaires sera entièrement équipé de "jouets», incluant deux hélicoptères et deux appareils de mobilité sous-marine (SEABOB) de sept passagers chacun, des embarcations pneumatiques (Zodiac) amphibies, un caisson de décompression pour la plongée, un véhicule tout terrain multi-passager (ces véhicules laissent des empreintes sur le sol), ainsi que tous les équipements récréatifs de ski nautique et de kayak de mer (Crystal, 2016). Ces équipements sous-entendent que les activités des passagers seront orientées vers des pratiques aventureuses avec tous les outils nécessaires pour exercer leur instinct de domination de la nature plutôt que d'encourager des expériences non agressives d'observation et d'harmonie avec l'environnement naturel. Ce développement, qui sera suivi par celui du transport commercial, illustre la banalisation de l'Arctique, nouveau terrain de jeux et d'affirmation des classes nanties. 


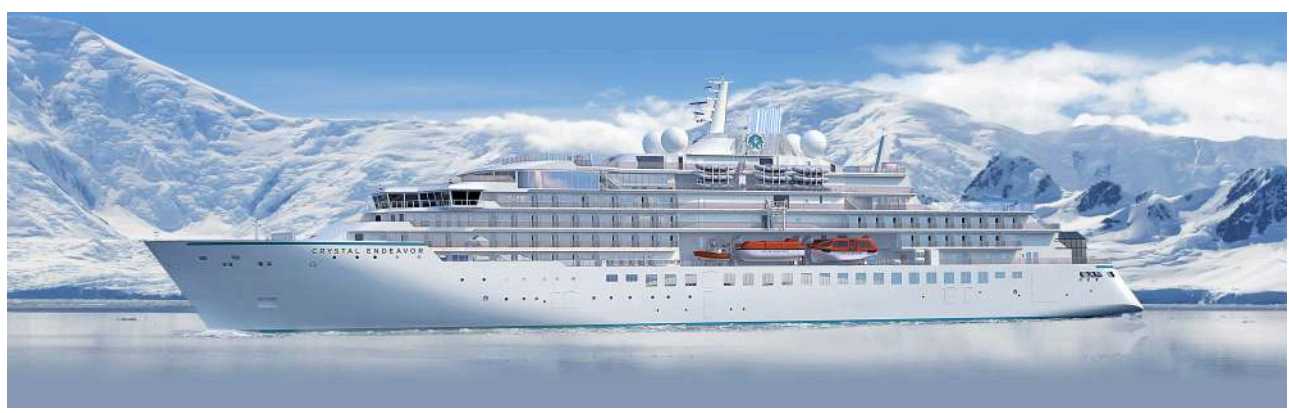

Le Crystal Endeavor - à la fine pointe du tourisme polaire

Source : Crystal (2018)

Et les Inuits dans tout cela ? Comme par le passé, ils assistent, relativement impuissants, au décloisonnement de leur territoire. Leurs propos, discrets et prudents, pèsent le chaud et le froid. S'ouvrir au monde menace leur tranquillité mais demeurer fermés sur leur territoire n'est plus une option viable. Car leur univers, affecté par les changements climatiques causés par le mode de vie des sociétés modernes, n'est plus le seul garant de leur avenir, jadis lié à une économie de subsistance. Les Inuits doivent eux aussi composer avec un univers «mondialisé » où le paysage, la faune, la flore et leur culture s'ajoutent aux ressources minières déjà en exploitation et qui financent, en partie, leur économie. La difficulté pour tous et chacun est de savoir où s'arrêter.

Pour l'État canadien, l'enjeu est de pouvoir s'imposer, en partenariat avec les Inuits, sur l'échiquier mondial, afin de défendre les intérêts d'un territoire aussi vaste qu'impitoyable mais au talon d'argile. Car faute de moyens, le Canada risque de voir lui échapper le contrôle du développement de son Arctique maritime. Celui-ci pourrait être pris en charge par un consortium d'acteurs, incluant des gouvernements et des entreprises non seulement étrangers au Canada, mais au monde arctique. Ce type de formule ne pourra être un compromis politico-économique viable que dans la mesure où les populations de l'Arctique font partie intégrante du processus consultatif et décisionnel afin que soient protégés leurs cultures et leurs modes de vie. Le décloisonnement contient les promesses d'un avenir économiquement plus confortable, mais écologiquement plus incertain.

Dans ce contexte, le tourisme polaire n'est plus qu'un phénomène socio-économique aux confins de l'imaginaire géographique. Il est aussi instigateur du changement. À la croisée des chemins, il peut décider du rôle qu'il veut jouer: soit il favorise la conservation du territoire, de ses habitants et de leurs ressources, comme il le fait, dans une certaine mesure actuellement ; soit il précède, par la banalisation de l'Arctique, son exploitation sans scrupule et sans fin. 


\section{BIBLIOGRAPHIE}

AHDR - Arctic Human Development Report, Arctic Human Development Report, Stefansson Arctic Institute: Akureyri, Islande, 2004.

Arctic Council, Arctic Marine Shipping Assessment 2009 Report, Arctic Council. Norvège, 2009. 194 pages.

BENNETT, Mia, « As the Arctic Melts, Singapore Rises to the Top », The Maritime Executive. https://www.maritime-executive.com/magazine/as-the-arctic-melts-singapore-rises-to-the-top, 2016. Consulté le 5 juin 2018.

BREED, Greg A; Cory J.D. MATTHEWS; Marianne MARCOUX; Jeff W. HIGDON; Bernard LeBLANC; Stephen D. PETERSON; Jack ORR; Natalie R. REINHART et Steven H. FERGUSON, "Sustained disruption of narwhal habitat use and behaviour in the presence of Arctic killer whales", PNAS, 2017, p. 1-6. https://www.researchgate.net/publication/ 251095008_The_Rise_of_Killer_Whales_as_a_Major_Arctic_Predator. Consulté le 5 juin 2018

BUTLER, R.W., « The concept of a tourist area life cycle of evolution: implications for management of resources ", Canadian Geographer, vol. 24, 1980, pp. 5-12. https:// www.numptynerd.net/uploads/1/2/0/6/12061984/butler_model_1980.pdf. Consulté le 5 juin 2018

CBC News, «Canada's Arctic rescuers not based in North », Canadian Broascasting Corporation, $1^{\mathrm{er}}$ novembre 2010. https://www.cbc.ca/news/canada/north/canada-s-arctic-rescuers-notbased-in-north-1.956604 Consulté le 5 juin 2018.

CBC News, "Outdated charts of Arctic waters could threaten ship safety: expert ", Canadian Broadcasting Corporation, 4 janvier 2008. https://www.cbc.ca/news/canada/north/outdatedcharts-of-arctic-waters-could-threaten-ship-safety-expert-1.763799. Consulté le 26 septembre 2018.

CHAN, Rarrah T.; Sarah A. BAILEY et Hugh J. MACISAAC, « La navigation dans l'Arctique: Passage Gratuit pour les espèces aquatiques envahissantes ", Méridien, Ottawa, Commission canadienne des affaires polaires, 2012, p. 7-12.

COLE, Dermot, « Outdated navigational charts are an Arctic Maritime disaster in the making ", Arctic Today, 10 septembre 2018. https://www.arctictoday.com/outdated-navigational-chartsarctic-maritime-disaster-making/. Consulté le 11 septembre 2018.

Crystal, « Crystal Cruises Announces The World's Largest Megayacht « Crystal Endeavor », Crystal, 20 mars 2016, https://www.crystalcruises.com/news/2016/03/20/crystal-cruisesannounces-the-world-s-largest-megayacht-crystal-endeavor. Consulté le 2 juin 2018 (lien supprimé depuis la date de consultation).

DAVISON, Janet, « Arctic mapping to make navigating Northwest Passage Safer », Anchorage Daily News, 14 octobre 2012. https://www.adn.com/arctic/article/arctic-mapping-make-navigatingnorthwest-passage-safer/2012/10/15/. Consulté le 25 juin 2018.

GEIGER, John et Alanna MITCHELL, Franklin's lost Ship - The Historic Discovery of HMS Erebus, Toronto, HarperCollins, 2015, 206 pages. 
Government of Nunavut (sans date), Tunngasaiji: A Tourism Strategy for Nunavummiut, Government of Nunavut. 54 pages. https://gov.nu.ca/sites/default/files/tourism-strategy-en-2-aug21web.pdf.

GRENIER, Alain A., « Conceptualisation du tourisme polaire - cartographier une expérience aux confins de l'imaginaire », Téoros, vol. 28, no. 1, 2009, p. 7-19.

GRENIER, Alain A., « Le tourisme de croisière », Téoros, Vol. 27, No. 2, 2008, p. 36-48.

GRENIER, Alain A., The Nature of Nature Tourism, Doctorat, Faculté de sociologie de l'Université de Laponie, Finlande, 2004, 480 pages.

GUÉRIN-LAJOIE, José; Alain CUERRIER et Linda SIEGWART COLLIER, The Caribou Taste Different Now - Inuit Elders Observe Climate Change, Nunavut Arctic College, 2016, 252 pages.

HASS, Christian et Stephen E.L. HOWELL, « Ice Thickness in the Northwest Passage », Geophysical Research Letters, No. 10.1002/2015GL065704, 2015, p. 7673-7680.

HEADLAND, R.K., « Transits of the Northwest Passage to End of the 2017 Navigation Season, Atlantic Ocean, Arctic Ocean, Pacific Ocean », Papier révisé. Scott Polar Research Institute, University of Cambridge, Royaume-Uni, 2018. https://www.spri.cam.ac.uk/resources/infosheets/ northwestpassage.pdf. Consulté le 31 mai 2018.

HINCHEY, Garrett, “Arctic cruise ship owners ordered to pay $\$ 469 \mathrm{~K}$ in costs for 2010 grounding”, CBC News, Canadian Broadcasting Corporation, 9 février 2017. https://www.cbc.ca/news/ canada/north/cruise-ship-clipper-adventurer-nunavut-judgement-1.3973937. Consulté le 30 juin 2018.

HOPPER, Tristin, « Declaring itself a 'near-Arctic state, 'China to build a 'Polar Silk Road' off Canada's north », National Post, 30 janvier 2018. https://nationalpost.com/news/canada/ declaring-itself-a-near-arctic-state-china-to-drive-a-polar-silk-road-off-canadas-north. Consulté le 30 juin 2018.

HOWELL, Stephen E.L.; Adrienne TIVY; John J. YACKEL et Steve McCOURT, « Multi-year sea-ice conditions in the western Canadian arctic archipelago region of the northwest passage: 1968-2006 ", Atmosphere-Ocean Journal, vol. 46, no. 2., 2007, p. 229-242.

HUEBERT, Rob, « Climate change and Canadian sovereignty in the Northwest Passage », pp. 383-399 dans Canadian Arctic Sovereignty and Security: Historical Perspectives, sous la direction de P. Whitney LACKENBAUER, Calgary Papers in Military and Strategic Studies, Occasional Paper no. 4, 2011.

Inuit Tapiriit Kanatami, Représentation cartographique. Tiré de Affaires autochtones et du Nord Canada. Gouvernement du Canada, 2015. https://www.aadnc-aandc.gc.ca/fra/ 1450197908882/1450197959844. Consulté le...

JAFARI, Jafar (1988), « Le système du touriste: Modèles socio-culturels en vue d'applications théoriques et pratiques», Loisir \& Société / Society \& Leisure, vol. 11, no. 1, 1988, p.59-80.

KELLY, Brenda P.; Andrew WHITELEY et David TALLMON, “The Arctic melting pot”, Nature, vol. 468, p. 891 (16 décembre 2010).

KHON, V.C.; I.I. MOKHOV; M. LATIV; V.A. SEMENOV et W. PARK, « Perspectives of Northern Sea Route and Northwest Passage in the twenty-first century", Climate Change. Volume 3-4, 2010, p. 757-768. 
Maritime Executive, «Liberia Issues Report into Investigation of Explorer Casualty », Maritime Executive, 2009. https://www.maritime-executive.com/article/2009-04-14-liberia-issues-reportinvestigation-explorer-casualty. Consulté le 5 juin 2018.

MACCANNELL, Dean, The Tourist: A New Theory of the Leisure Class, Berkeley, University of California Press, 1976, r. 1989, 280 pages.

MARSH, J. et S. STAPLE, « Cruise tourism in the Canadian Arctic and its implications", pp. 63-72, dans . Polar tourism: Tourism and the Arctic and Antarctic regions, sous la direction de C. Michael HALL et Margaret E. JOHNSTON, Chichester, John Wiley and Sons, 1995, 329 pages.

MASON, Peter, « Tourism Codes of Conduct in the Arctic and Sub-Arctic Region », Journal of Sustainable Tourism, Vol. 5, No. 2, 2010, p. 151-165.

NBS - Nunavut Bureau of Statistics, « StatsUpdate», Nunavut Bureau of Statistics, 2018. https:// www.gov.nu.ca/sites/default/files/

nunavut_and_canada_population_estimates_statsupdate_first_quarter_2018.pdf. Consulté le 18 septembre 2018.

Nunavut Tourism, « Nunavut Visitor Exit Survey 2015 - Final Report », Nunavut Tourism, Iqaluit, 2016, 88 pages.

PHARAND, Donat, « The Arctic Waters and the Northwest Passage : A Final Revisit », Ocean Development \& International Law, Vol. 38, No. 1-2, 2007, p. 3-69.

STEWART, Emma J. et D. DRAPER, « The sinking of the MS Explorer : Implications for Cruise Tourism in Arctic Canada », Arctic Journal, Vol. 61, no. 2, 2008, p. 224-228.

Von SCHEEL, Elise, « Black market animal smuggling is booming in Canada », CBC News, Canadian Broadcasting Corporation 26 juin 2018. https://www.cbc.ca/news/politics/black-market-animalsmuggling-canada-1.4720102. Consulté le 26 juin 2018.

WISZ, M. S.; O. BROENNIMANN; P. GRØNKJAER; P.R. MØLLER; S.M. OLSEN; D. SWINGEDOUW; R.B. HEDEHOLM; E.E. NIELSEN; A. GUISAN et L. PELLISSIER, « Arctic warming will promote AtlanticPacific fish interchange ", Nature Climate Change, vol. 5, 2015, p. 261-265.

\section{Communications personnelles}

NDEDT - Nunavut Department of Economic Development and Transportation (2018), Communications personnelles. Le 25 septembre 2018.

\section{NOTES}

1. L'auteur de l'article a lui-même livré une communication devant les délégués, sur les enjeux du tourisme dans le passage maritime convoité.

2. Pour une discussion sur le concept de Nord, voir AHDR (2004) et Grenier (2009).

3. Il faut prendre ces données à titre indicatif seulement, l'auteur de la présente étude étant en mesure d'identifier plusieurs croisières absentes de la liste de Headland (2018).

4. Le tourisme de masse ne doit pas être perçu dans le sens péjoratif du terme. Le mot "masse » signifie ici que la destination accepte des quantités importantes de visiteurs, de façon continue tout au long de l'année. Lorsqu'il est bien géré, le tourisme de masse peut être bénéfique pour les communautés, en offrant un revenu stable sur une partie de l'année.

5. Le nombre de passagers est calculé à partir du nombre de places disponibles à bord des navires. Les données ne garantissent pas que toutes les places à bord des navires aient été 
vendues. Pour le nombre de passagers en 2008, données du Government of Nunavut (sans date: 18). Pour le nombre de passagers en 2015, Nunavut Tourism (2016:10).

\section{RÉSUMÉS}

Stimulés par l'impact des changements climatiques sur l'Arctique, plusieurs groupes d'intérêt (États et armateurs) voient dans le passage du Nord-Ouest, le rêve d'une route navigable entre l'Europe et l'Asie au nord du continent nord-américain. Faute de moyens, l'État canadien tarde à développer les infrastructures nécessaires à la commercialisation du passage, remettant en cause sa capacité à assumer sa souveraineté sur le passage convoité. Pendant ce temps, se développe depuis trois décennies un tourisme d'aventures maritimes dans ce milieu caractérisé par des conditions géo-climatiques et socio-économiques extrêmes.

Le territoire hôte de ce phénomène se nomme Nunavut - "notre terre ", en inuktitut. Le tourisme «polaire » qui s'y développe se justifie par l'intérêt du public pour la nature (faune, flore, paysage) des régions polaires, une curiosité pour le peuple inuit qui habite cette terre, et un désir à peine caché par les touristes d'atteindre ces latitudes extrêmes, longtemps maudites par les récits tragiques des explorateurs.

Outre les enjeux de navigation auxquels sont également confrontés les armateurs, l'industrie du tourisme de croisière polaire fait face à des défis qui lui sont spécifiques. D'une part, l'éparpillement des attraits touristiques sur le Nunavut - territoire de $2000000 \mathrm{~km}^{2}$ pour une population de 38000 habitants (NBS, 2018) - représente un casse-tête organisationnel majeur pour les voyagistes. S'ajoutent à cela l'absence des infrastructures nécessaires (aéroports, ports et hébergement) pour accueillir les visiteurs et la prise en compte de leur sécurité. Ce développement interpelle aussi les Inuits quant à leur participation à cette activité économique, à la gestion et à la protection de leur patrimoine naturel et culturel.

Située à la croisée de la géographie et de la sociologie, cette étude montre comment l'industrie touristique, par son exploration des eaux arctiques, contribue en tant qu'éclaireur au décloisonnement d'un territoire maritime qu'elle cherchait, a priori, à protéger.

Stimulated by the impact of climate change on the Arctic, several interest groups (states and shipowners) see the Northwest Passage as a dream route to navigate between Europe and Asia at the far North of the North American continent. Due to a lack of resources, the Canadian government is delaying the development of the infrastructures necessary to commercialize the passage, jeopardizing its ability to assume sovereignty over the coveted passage. Meanwhile, for the past three decades, maritime adventure tourism has been developing in this environment characterized by extreme geo-climatic and socio-economic conditions.

Nunavut - "our land" in Inuktitut - is the host territory for this phenomenon. The "polar" tourism that is developing there is justified by the public's interest in the nature (fauna, flora, landscape) of the polar regions, a curiosity for the Inuit people who inhabit this land, and a desire barely hidden by tourists to reach these extreme latitudes, long cursed by the tragic stories of explorers.

In addition to the navigational challenges shipowners also face, the polar cruise tourism industry encounters other issues, specific to its case. On the one hand, the scattering of tourist attractions in Nunavut - a territory of $2,000,000,000 \mathrm{~km} 2$ with a population of 38,000 (NBS, 2018) - represents a major organizational challenge for tour operators. In addition, there is a lack of the necessary 
infrastructures (airports, harbours and accommodation) to welcome visitors and to take their safety into account. This development also challenges the Inuit people as to their role in this economic activity as well as in the management and protection of their natural and cultural heritage.

Located at the crossroads of geography and sociology, this study shows how the tourism industry, through its exploration of Arctic waters, scout-like, contributes to the opening of a maritime territory that it sought, initially, to protect.

Estimulados pelo impacto das mudanças climáticas no Ártico, vários grupos de interesse (Estados e armadores) consideram a passagem do Noroeste como um sonho de estrada navegável entre a Europa e a Ásia, no norte do continente norteamericano. Na falta de recursos, o Estado canadense demora em desenvolver as infraestruturas necessárias para a comercialização da passagem, o que alimenta as dúvidas sobre sua capacidade em assumir sua soberania nessa passagem. Enquanto isso, há três décadas que cresce um turismo de aventuras marítimas, nesse meio caracterizado por condições geo-climáticas e socio-econômicas extremas.

O território evocado se chama Nunavut - "nossa terra", em inuktitut; o turismo "polar" que se desenvolve ali radica no interesse do público pela natureza (fauna, flora, paisagens) das regiões polares, numa curiosidade para o povo inuit que vive nessas terras, e num desejo mal disfarçado de atingir essas latitudes extremas, que durante muito tempo pareciam malditas nos relatos trágicos dos exploradores.

Além das questões de navegação, que os armadores devem também enfrentar, a indústria do turismo de cruzeiro polar responde a outros desafios que lhe são específicos. Por um lado, a dispersão das atrações turísticas no Nunavut - território de $2000000 \mathrm{~km}^{2} \mathrm{com}$ uma população de 38000 habitantes (NBS, 2018) - representa um quebra-cabeça organizacional para os operadores turísticos. A isso se some a ausência de infraestruturas necessárias (aeroportos, portos e alojamentos) para receber os visitantes e garantir sua segurança. o desenvolvimento do turismo leva também os Inuits a se interrogar sobre o fato de participar ou não a essa atividade econômica, assim comon sobre a gestão e a proteção de seu patrimônio natural e cultural.

Numa encruzilhada entre geografia e sociologia, este estudo assinala como a indústria turística, tendo um papel precursor através da exploração da navegação no Ártico, contribui para a abertura de um território marítimo que, num primeiro momento, ela tentava proteger.

\section{INDEX}

Mots-clés : tourisme, navigation, enjeux, Arctique, Passage du Nord-Ouest, Nunavut

Keywords : tourism, navigation, issues, Arctic, Northwesp Passage, Nunavut

Palavras-chave : turismo, navegação, desafios, Ártico, Passagem do Noroeste, Nunavut

\section{AUTEUR}

\section{ALAIN ADRIEN GRENIER}

Groupe de recherche et d'intervention tourisme, territoire et société (GRITTS). Département d'études urbaines et touristiques. École des sciences de la gestion. Université du Québec A Montréal (ESG UQAM)Grenier.alain-adrien@uqam.ca 